\title{
Event shapes for hadronic final state: experimental review
}

\author{
V.A. Okorokov ${ }^{\text {a }}$
}

National Research Nuclear University MEPhI,

Kashirskoe Shosse 31, 115409 Moscow, Russian Federation

date May 24, 2011

\begin{abstract}
Analysis is presented for first moments of collective observable distributions in two-jet events for various interaction types and for wide initial energy range. These observables include sphericity, thrust, components of transverse particle momentum, alignment and planarity. Database of experimental results created in the framework of the paper includes data for all interactions. Energy dependencies of average values for collective observables excepting of transverse momentum components show universal behavior for various interactions. Thus energy dependence for these parameters supposes the descriptions by functions which are universal for different interaction types. Particle transverse momentum as well as it's components increase faster for $e^{+} e^{-}$interaction with growth of $\sqrt{s}$, than that for other interactions. Empirical analytical functions are suggested for description of energy dependence for all collective observables under study with exception of infrared-stable thrust variable. Energy dependence for average thrust is compared with QCD predictions included perturbative part and analytical phenomenological corrections which account for nonperturbaive effects. Dispersive model and single dressed gluon approximation are considered for description of energy dependence of first moment of thrust distribution and estimation of strong coupling constant for various interactions as well as for joint sample. The dispersive model allow to describe average thrust vs initial energy in wide range of $\sqrt{s}$ down to strongly non-perturbative domain $\sqrt{s} \sim 2-3 \mathrm{GeV}$ at qualitative level at least. Study of event shape observables allows to obtain estimations of $\alpha_{S}\left(M_{Z}\right)$ which are in reasonable agreement both with world average value and with results extracted in the framework of other methods. Using suggested analytic approximation functions estimations of values of collective parameters under study have been obtained for present and future facilities. In $\mathrm{TeV}$ energy domain average values of collective observables either depend on $\sqrt{s}$ weakly or do not depend on initial energy at all within errors. Thus, the $\mathrm{TeV}$ scale can be considered as an estimation of the low bound of asymptotic region for traditional collective parameters. Usually, multiplicity dependence of collective observables under considered agree with power function in energy domain $\sqrt{s}<12 \mathrm{GeV}$ at qualitative level at least. Behavior of sphericity vs multiplicity and comparison of experimental results with model calculations allow to suggest that the universal estimation of the low energy boundary for experimental appearance of event jet structure in multiparticle production processes is $\sqrt{s_{c}} \sim 3 \mathrm{GeV}$.
\end{abstract}

PACS. 13.87.-a Jets in large- $Q^{2}$ scattering - 12.38.-t Quantum chromodynamics

\section{Introduction}

In various interactions and in wide initial energy domain final state hadrons appear predominantly in collimated bunches heading in roughly the same direction within a certain opening angle. These bunches are generically called jets. Hadronic jets is one of the most spectacular phenomena in particle physics.

Since we are not able to directly measure colored objects (quarks or gluons), but only hadrons and their decay products, a central issue for every experimental test of quantum chromodynamics (QCD) is establishing a correspondence between observables obtained at the partonic and the hadronic level. Hadronic jets provide a window

\footnotetext{
a e-mail: VAOkorokov@mephi.ru; Okorokov@bnl.gov
}

into the fundamental workings of QCD, since they contain within themselves the signatures of QCD at both weak and strong coupling. Jets therefore test our understanding of QCD over a wide range of scales [1]. Jets are used also for identifying the hard partonic structure of decays of massive particles like top quarks. In order to map observed hadrons onto a set of jets, one uses a jet definition. Unfortunately formulation of universal and quantitative definition of hadronic jet for various experiments is a difficult problem. To a first approximation at enough high energies, a jet can be thought of as a hard parton that has undergone soft and collinear showering and then hadronization. Good jet definitions have to be infrared and collinear safe, simple to use in theoretical and experimental contexts, applicable to any type of inputs (parton or hadron momenta, charged particle tracks, and/or en- 
ergy deposits in the detectors) and lead to jets that are not too sensitive to non-perturbative effects [2]. The review of investigations of jet definitions is given in [3] (for $e^{+} e^{-}$annihilation) and in $[4,5]$ (for hadronic - pp / $\bar{p}$ p collisions).

On the one hand, one can study observables that depend on specifying the actual number of jets in the final state by defining a jet algorithm, which sets criteria for what constitutes a jet. There are two main classes of such algoritms: cone algorithms, extensively used at hadron colliders, and sequential recombination algorithms, more widespread at $e^{+} e^{-}$and $e$ p colliders. On the other hand, one can extract much useful information about the structure of the final state from simpler observables called event shapes or collective variables, which do not depend on a jet algorithm, but are simple functions of the momenta of all final state particles included in jet analysis.

Event shapes are used for many purposes. Some collective characteristics can be calculated perturbatively in terms of QCD colored objects and compared with hadronic final state measurements at high energies [6]. The LEP experiments and their high quality data have prompted much theoretical progress in the understanding of QCD radiation and in the development of appropriate tools. This includes Sudakov resummation, renormalon resummation and parametrization of power corrections [7]. At the same time, experimental uncertainties and non-perturbative effects are small in the regime of high transverse momenta, which makes possible precision tests of perturbative QCD and the extraction of the strong coupling constant. On the other hand, collective observables are directly sensitive to the hadronization process, providing an opportunity to learn more about the confinement mechanism from experiment [7]. Understanding the nature of hadronisation is a challenge, which is crucial important for all high energy experiments involving hadrons [8]. Thus the study of collective observables in hadronic collisions and for wide energy domain seems the important task and promising tool for investigation of problem of confinement, hadronization mechanism and corresponding analytical models. At the same time that hadronic jets provide insights into QCD, they are key elements in signatures for new physics beyond the Standard Model. Some traditional event shape observables can be used for search of such signatures. The detail investigations of the sphericity and thrust distributions are promising tools for sharp and robust discrimination between the SUSY and some models with large extra dimensions which should be utilisable either at ILC or at CLIC wherever these scenarios become accessible [9]. At a hadronic colliders such as the LHC, most interesting processes involve final states with jets of hadrons. The data available to define and reconstruct the jets are energy depositions in the calorimeter and charged particle tracks. Ideally one can use this experimental data to identify an underlying hard scattering event that involved QCD partons in the weakly coupled high energy regimes or, alternatively, heavy standard model particles such as top quarks or newly produced nonstandard model particles. Distinguishing the former from the latter is of course crucial to our ability to find and analyze new physics at the LHC [10]. Thus traditional collective variables can be useful for search of some exotic events in hadronic collisions at (ultra)high energies. As expected ordinary QCD events can be distinguished from events involved decays of new particles because values of some event shapes will be higher (or closer to the spherical limit) for latter case than that in the former one. In particular, higher sphericity of an event in relation to any other event of interactions described by the Standard Model is of the general regularities for multidimensional black hole production at accelerators [11]. But one need to emphasize that the challenge of calculating QCD background events in the hadron collider environment of the RHIC, Tevatron or the LHC remains formidable so far.

This paper focuses at experimental study of event shapes namely. At present the pp colliders RHIC and LHC as well as Tevatron allows to study the geometry of final state in new energy ranges at high precisions. Taking into account importance of study of hadronic interactions but not only collisions with lepton beams this paper pais attention, especially, to event shapes in hadron-hadron and hadron-nuclear reactions.

The paper is organized as follows. In Sec.2, definitions of collective variables under study are described. Database created in the framework of the paper is shown and discussed in Sec.3. The Sec.4 devotes to discussion of energy dependence of collective variables, comparison it with various approximations and extracted estimations of strong coupling constant. Section 5 demonstrates a multiplicity dependence of jet characteristics and in Sec.6 some final remarks are presented.

\section{Observables in analysis of hadronic final state properties}

Event shape variables are functions of the four momenta in the hadronic final state that characterize the topology of an event's particle / energy flow. They are sensitive to QCD radiation (and correspondingly to the strong coupling) insofar as gluon emission changes the shape of the particle / energy flow [2]. The relationship of pattern of collective particle / energy flow with measured parameters (momenta) of final state particles can be described by momentum tensor $\Lambda^{\alpha \beta}$ which is defined generally in the following way:

$$
\Lambda^{\alpha \beta}=\sum_{i=1}^{N} w_{i} p_{\alpha}^{i} p_{\beta}^{i}, \quad \alpha, \beta=x, y, z .
$$

Here $N$ is the number of particles separated for collective analysis in event, $w_{i}$ - some weight of $i^{\text {th }}$ particle. Both the collective flow of particles and energy can be investigated at choosing the suitable $w_{i}$. One need to emphasize that the form of the momentum tensor varies for different papers (see, for example, $[12-16]$ ).

One of the most important and global properties of hadrons produced in various collisions in wide energy range 
is their jet nature which can be characterized by the some collective observable. The sphericity $(S)$ and thrust $(T)$ are the most popular and widely used variables for study of jet behavior of the secondary particles. The sphericity is defined as the following $[12,13]$ :

$$
S=\min _{\boldsymbol{n}} S(\boldsymbol{n})=\frac{3}{2} \min _{\boldsymbol{n}} \frac{\sum_{i=1}^{N} p_{\perp i}^{2}}{\sum_{i=1}^{N} \boldsymbol{p}_{i}^{2}} .
$$

Here $p_{\perp i}^{2}$ is the transverse momentum of the $i^{\text {th }}$ particle with respect to the sphericity axis, which is chosen in such a way that the $\operatorname{sum} \sum_{i=1}^{N} p_{\perp i}^{2}$ with respect to it is minimal; $\boldsymbol{p}_{i}$ is the momentum of the particles in the center-of-mass system, and the sum extends over all particles separated for jet analysis. Since sphericity is quadratic in the momenta it cannot be calculated in perturbation part of the QCD, however it has remained a popular jet measure because it can be easily determined [17]. A measure of the event structure that uses the linear momenta is the thrust $[18-20]$

$$
T=\max _{\boldsymbol{n}} T(\boldsymbol{n})=\max _{\boldsymbol{n}} \frac{\sum_{i=1}^{N}\left|p_{\| i}\right|}{\sum_{i=1}^{N}\left|\boldsymbol{p}_{i}\right|} .
$$

The thrust axis being chosen to maximize $\sum_{i=1}^{N}\left|p_{\| i}\right|$, the sum of the components of the momenta parallel to the thrust axis of all particles in an event selected for jet analysis.

Sphericity tensor $S^{\alpha \beta}$ can be derived as special case of general form (11) at $\forall i: w_{i}=$ const. Usually constant is chosen equal to unit or as follow const ${ }^{-1}=\sum_{i=1}^{N} \boldsymbol{p}_{i}^{2}$. The present work is used the first case of constant and the matrix of sphericity tensor is the following:

$$
S^{\alpha \beta}=\sum_{i=1}^{N} p_{i}^{\alpha} p_{i}^{\beta}, \quad \alpha, \beta=x, y, z .
$$

Because the sphericity tensor uses the momenta of particles quadratically, the high-momentum particles in an event will contribute more strongly to observables derived from this tensor than to those which use the momenta linearly [21]. To eliminate false effects which may be caused by leading particles, the reduced-momentum matrix [22] can be also used to study the configurations of inelastic collisions.

Study of shape of hadronic final state in various interactions is carried out in the system of coordinates of the "principle axes" of the event, which are the symmetry axes for a given configuration of secondary-particle momentum vectors (Fig 1). This coordinate system has some special advantages for a more detailed study of the configuration of events in 3-momentum space. Conversation of this coordinate system was accomplished by standard procedure of diagonalization of a matrix (4). Let $\boldsymbol{n}_{1}, \boldsymbol{n}_{2}$, and $\boldsymbol{n}_{3}$ be the unit eigenvectors of the tensor (4) associated with the eigenvalues $\lambda_{j}$, which are ordered such that $\lambda_{1} \geq \lambda_{2} \geq \lambda_{3}$ and $\lambda_{\sum} \equiv \sum_{i=1}^{3} \lambda_{i}$. For this choice the vector $\boldsymbol{n}_{1}$ determines the direction of the greatest extent of the event in momentum space; $\boldsymbol{n}_{2}$ determines the direction of greatest extent in the plane perpendicular to $\boldsymbol{n}_{1}$, and $\boldsymbol{n}_{3}$ determines the direction of the greatest compression in this plane. The axis coincided with direction of eigenvector $\boldsymbol{n}_{1}$ is jet axis in the assumption that the certain event show two-jet structure. The diagonal elements of the matrix (4) represent the sum of the squares of the components of the secondary-particle momenta in the coordinate system of the principal axes. If the diagonal elements are related as $\lambda_{1} \sim \lambda_{2} \sim \lambda_{3}$, then the event is spherically symmetric; if $\lambda_{1} \gg \lambda_{2}, \lambda_{1} \gg \lambda_{3}$, and $\lambda_{2} \sim \lambda_{3}$, the event has cyllindrical symmetry; if $\lambda_{1} \sim \lambda_{2}$ and $\lambda_{1}, \lambda_{2} \gg \lambda_{3}$, then the event is planar, recalling a disk in shape [23]. Forms of events at various values of traditional collective observables are presented, for example, in [20].

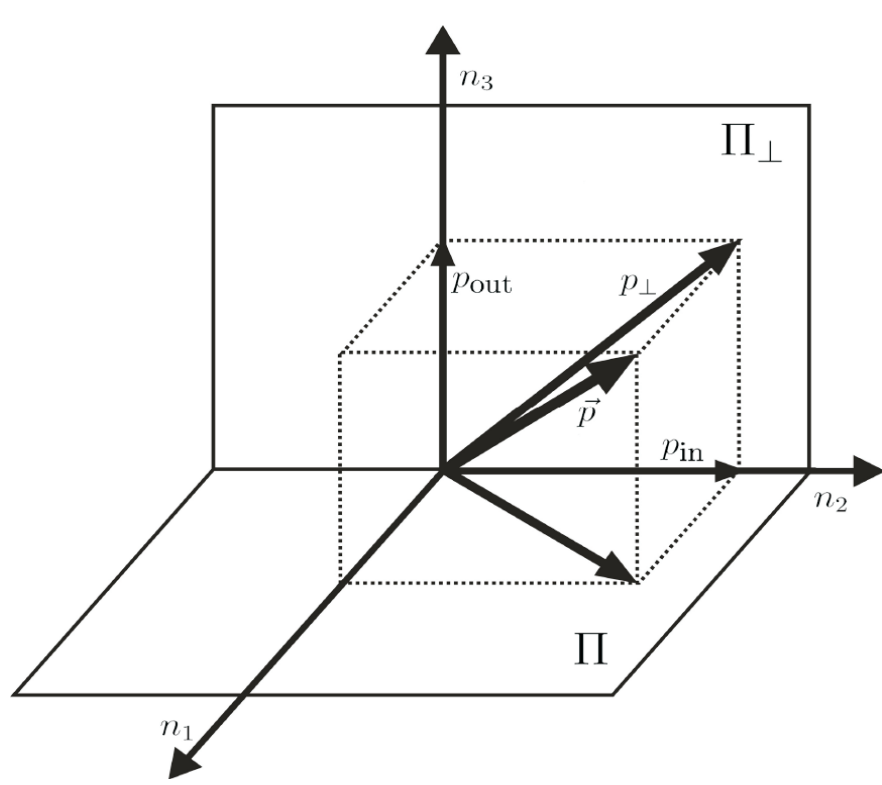

Fig. 1. System of coordinates of the "principle axes" $\left(\boldsymbol{n}_{1}, \boldsymbol{n}_{2}, \boldsymbol{n}_{3}\right)$. Also longitudinal plane $(\Pi)$ containing jet axis event plane, transverse plane $\left(\Pi_{\perp}\right)$ and components of particle momentum $\boldsymbol{p}$ in transverse plane are shown in the system of coordinate.

Historically sphericity and thrust are considered as main traditional variables characterized the geometry of final hadronic state. There are several additional collective observables derived from the sphericity tensor. In particular, $p_{\text {in }}^{2}$ and $p_{\text {out }}^{2}$ are, respectively, the squares of particle transverse momenta in and out of the event plane (Fig:1). One of the versions for definitions of collective variables via eigenvalues of sphericity tensor $S^{\alpha \beta}$ are shown in the Table 1 
Table 1. Event shape observables

\begin{tabular}{lc}
\hline \multicolumn{1}{c}{ Observable } & Definition \\
\hline sphericity $(S)$ & $3\left(\lambda_{2}+\lambda_{3}\right) / 2 \lambda_{\Sigma}$ \\
$p_{\text {in }}^{2}$ & $\lambda_{2} / N$ \\
$p_{\text {out }}^{2}$ & $\lambda_{3} / N$ \\
alignment (A) & $\lambda_{2} / \lambda_{1}$ \\
planarity (P) & $\lambda_{3} / \lambda_{2}$ \\
\hline
\end{tabular}

According to definitions (2) and (3) $S \rightarrow 1$ and $T \rightarrow$ 0.5 for events with large number of the secondary particles distributed isotropically in phase space; $S \rightarrow 0$ and $T \rightarrow 1$ if two separated and narrow collimated particle groups are produced in interaction process which are directed to the opposite sides. Magnitudes of components $p_{\text {in }}^{2}$ and $p_{\text {out }}^{2}$ depend on a choice of a reference plane in a longitudinal direction, i.e. a plane contained jet axis (plane $\Pi$ at Fig (1) while absolute value of square of particle transverse momentum with respect to the jet axis $p_{\perp}^{2}=p_{\text {in }}^{2}+p_{\text {out }}^{2}$ does not depend on a choice of this plane (Fig[1). The detailed review of the experimental results obtained for choice as (longitudinal) scattering plane the plane formed by the two beams and the trigger particle is presented, for example, in [24]. Events with greater alignment are characterized by smaller value of parameter A, for strongly planar events is valid the relationship $\mathrm{P} \ll 1$.

Symbol $Y$ denotes below the generic event shape variable from the set under study. The $n^{\text {th }}$ moment of the distribution of an event shape parameter $Y$ is defined by

$$
\left\langle Y^{n}\right\rangle=\int_{0}^{Y_{\max }} d Y Y^{n} \frac{1}{\sigma_{\mathrm{tot}}} \frac{d \sigma}{d Y}, \quad n=1,2, \ldots
$$

Here $Y_{\max }$ is the kinematically allowed upper limit of variable $Y, \sigma_{\text {tot }}-$ total hadronic cross section. The present paper focuses on the first moments of distributions of collective variables.

\section{Experimental data sample}

Experimental results for event shape variables are considered for all interaction types and for total available energy range in the paper. One need to stress that although event shape of hadronic final state is studied for a long time there is no standard database for collective characteristics. The experimental data are presented in the HEPDATA [25] and we made attempt to collect results for wide set of collective observables. Table 2 shows corresponding facilities and their experiments. Results from latest paper of the experiment are included for given collective parameter at certain energy.

To average data from different experiments at equal or, at least, (very) close energies weighted procedure is used. We assume that measurements of given quantity are uncorrelated in different experiments, and calculate a weighted average as

$$
\bar{Y}=\frac{\sum_{i} \xi_{i}\langle Y\rangle_{i}}{\sum_{i} \xi_{i}}
$$

where $\langle Y\rangle_{i}$ is the average value of given observable reported by the $i^{\text {th }}$ experiment, $\xi_{i}$ is the weight of the $i^{\text {th }}$ experiment which is equal statistics / luminosity used for study of given observable in the experiment, and the sums run over the all experiments at certain energy. Table 3 shows energy values and corresponding experiments whose results were averaged for some given collective observable. The notation $\sqrt{s}$ is used for all interaction types but one need to stress that $Q$ (absolute value of momentum transfer) and $W$ (invariant mass of final hadronic state) imply as initial energy parameter for DIS experiments at HERA and for experiments with (anti)neutrino beams respectively.

It would be stressed that the common basis, in particular, adequate initial energy scale needs for correct comparison between various ways of producting multihadronic final states [74]. Identification of the correct initial energy scale, especially, for hadronic interactions, seems the nontrivial task. There are different methods for definition of energy scale in hadronic reactions (see, for example, [60, $63,66]$ ) but universal scheme is absent so far. Detail studies in the framework of the paper show that $\sqrt{s}$ can be used as an energy scale in hadronic collision together with some specific additional cuts for event / particle selection (for example, reducing of influence of leading particle etc.). Comparison of average values of different collective observables will show reasonable agreement for various interactions at such choice of energy scale for hadronic interaction (see below).

Also averaging has been made on Breit variable $x$, beam energy for some DIS experiments and on different measured kinematic ranges for some hadronic interactions in order to exclude the possible influence of additional kinematic cuts on the collective characteristic values. Errors of experimental points include available clear indicated systematic errors added in quadrature to statistical ones. One need to emphasize that the most of data for main collective parameters (sphericity and thrust) are published results. On the other hand the some values of the above parameters as well as for other observables are estimations which were obtained on the basis of mean values of eigenvalues of sphericity tensor or at experimental distributions of $p_{\text {in }}, p_{\text {out }}$. Obviously, such approach allows to get only rough estimations of true average values. But on the other hand the smooth experimental dependencies will be presented below and corresponding discussion show that estimations obtained by this method seem reasonable for any collective parameters under study and allow to extend energy domain significantly. Also the values of main collective observables for special cases as well as, for example, sphericity and thrust values for quarkonium states $[29,30]$ or thrust values in the very narrow energy ranges at searching for top quark [36] are not shown at the global fits below. 
Table 2. Facilities and experiments

\begin{tabular}{|c|c|c|c|}
\hline Facility & Location & Experiment / Detector & Refs. \\
\hline \multicolumn{4}{|c|}{ 1. $e^{+} e^{-}$annihilation } \\
\hline SPEAR & SLAC Stanford & SLAC-LBL & {$[13]$} \\
\hline \multirow[t]{3}{*}{ PEP } & & DELCO & {$[26]$} \\
\hline & & HRS & {$[27]$} \\
\hline & & Mark II & {$[28]$} \\
\hline \multirow[t]{2}{*}{ DORIS } & DESY Hamburg & PLUTO & {$[17,29]$} \\
\hline & & $\mathrm{NaI}$ & {$[30]$} \\
\hline \multirow[t]{5}{*}{ PETRA } & & CELLO & [31] \\
\hline & & JADE & {$[32-34]$} \\
\hline & & MARK-J & {$[35,36]$} \\
\hline & & PLUTO & {$[17,37]$} \\
\hline & & TASSO & {$[38,39]$} \\
\hline \multirow[t]{2}{*}{ TRISTAN } & KEK Tsukuba & AMY & {$[21]$} \\
\hline & & TOPAZ & {$[40]$} \\
\hline \multirow[t]{2}{*}{ SLC } & SLAC Stanford & Mark II & {$[41]$} \\
\hline & & SLD & {$[42]$} \\
\hline \multirow[t]{4}{*}{ LEP } & CERN Geneva & ALEPH & [43] \\
\hline & & DELPHI & {$[44]$} \\
\hline & & L3 & {$[45]$} \\
\hline & & OPAL & {$[33,46]$} \\
\hline \multicolumn{4}{|c|}{ 2. interactions with lepton beams } \\
\hline SPS & CERN Geneva & $4 \mathrm{~m} \mathrm{BC}(\mathrm{BEBC})$ & {$[47]$} \\
\hline PS & FNAL Batavia & $4.6 \mathrm{~m} \mathrm{BC}$ & {$[48]$} \\
\hline \multirow[t]{2}{*}{ HERA } & DESY Hamburg & H1 & {$[49-52]$} \\
\hline & & ZEUS & {$[53]$} \\
\hline \multicolumn{4}{|c|}{ 3. hadron-hadron collisions } \\
\hline AGS & BNL Upton & $2 \mathrm{~m} \mathrm{BC}$ & {$[54]$} \\
\hline \multirow[t]{4}{*}{ PS } & CERN Geneva & $0.8 \mathrm{~m}$ and $2 \mathrm{~m} \mathrm{BCs}$ & {$[54]$} \\
\hline & & $2 \mathrm{~m} \mathrm{BC}$ & {$[22]$} \\
\hline & & $1.5 \mathrm{~m}, 2 \mathrm{~m}$, and $4 \mathrm{~m} \mathrm{BCs}$ & {$[55]$} \\
\hline & & $4 \mathrm{~m} \mathrm{BC}$ & {$[56]$} \\
\hline \multirow[t]{4}{*}{ ISR } & & CMOR & {$[57]$} \\
\hline & & NA5 & {$[58]$} \\
\hline & & NA23 & {$[59]$} \\
\hline & & $\mathrm{SFM}$ & {$[60]$} \\
\hline $\mathrm{S} \overline{\mathrm{p} p S}$ & & UA2 & {$[61]$} \\
\hline LHC & & CMS & {$[62]$} \\
\hline \multirow[t]{2}{*}{ PS } & FNAL Batavia & IHSC & {$[63]$} \\
\hline & & LAFMS & {$[64]$} \\
\hline Tevatron & & $\mathrm{CDF}$ & {$[65]$} \\
\hline \multirow[t]{3}{*}{$\mathrm{U}-70$} & IHEP Protvino & 2m BC JINR ("Ludmila") & {$[23,66]$} \\
\hline & & $2 \mathrm{~m} \mathrm{BC}$ & {$[67]$} \\
\hline & & 4.5m BC ("Mirabelle") & {$[68,69]$} \\
\hline \multicolumn{4}{|c|}{ 4. hadron-nuclear interactions } \\
\hline PS & ITEP Moscow & $1 \mathrm{~m}$ and $2 \mathrm{~m} \mathrm{BCs}$ & {$[70,71]$} \\
\hline $\mathrm{U}-70$ & IHEP Protvino & 2m BC JINR & {$[72]$} \\
\hline SPS & CERN Geneva & $\mathrm{BEBC}$ & {$[47]$} \\
\hline $\mathrm{PS}$ & FNAL Batavia & $\mathrm{E} 260$ & {$[73]$} \\
\hline
\end{tabular}


Table 3. Energy and corresponding experiments for averaging

\begin{tabular}{|c|c|}
\hline$\sqrt{s}, \mathrm{GeV}$ & Experiments \\
\hline \multicolumn{2}{|c|}{ 1. $e^{+} e^{-}$annihilation } \\
\hline 9.4 & NaI, PLUTO \\
\hline $12.0 ; 13.0 ; 17.0 ; 27.3 ; 30.6$ & MARK-J, PLUTO, TASSO \\
\hline 14.0 & JADE, TASSO \\
\hline 22.0 & JADE, MARK-J, PLUTO, TASSO \\
\hline 29.0 & DELCO, HRS, Mark II \\
\hline 35.8 & CELLO, JADE, MARK-J, TASSO \\
\hline 38.3 & JADE, MARK-J \\
\hline 43.6 & CELLO, JADE, TASSO \\
\hline$M_{Z}$ & ALEPH, DELPHI, L3, Mark II, OPAL, SLD \\
\hline $133.1-206.1$ & ALEPH, DELPHI, L3, OPAL \\
\hline \multicolumn{2}{|c|}{ 2. interactions with lepton beams } \\
\hline $3.2 ; 3.9 ; 5.0 ; 6.5 ; 10.6$ & BEBC, FNAL BC \\
\hline $9.3 ; 13.3 ; 18.3$ & H1, ZEUS \\
\hline $11.0 ; 15.2 ; 17.7 ; 22.6 ; 29.7$ & \\
\hline $36.8 ; 42.6 ; 58.9 ; 82.3 ; 116.3$ & \\
\hline
\end{tabular}

As known definitions of collective observable may vary from one experiment to other and depend on interaction type and energy domain under study (for example, there are several definitions for thrust analogies). But in the framework of this paper we have tried (if it was possibly) either to estimate the average values of collective parameter or recalculate they in according with the definitions shown in Table1 Moreover, for thrust we chose in original papers the definition of the variable which is most close to the (3) both in mathematical and physical meaning for all interactions in high energy domain.

\section{Energy dependence}

On Fig 2 dependencies of average values of collective observable sphericity (a) and thrust (b) on collision energy are shown. Experimental results and the estimations for collective characteristics under study are taken from a database presented in Table 2. As seen from Fig 2 general tendency for interactions of various types is observed both for sphericity (a) and for thrust (b) at a qualitative level, moreover the discrepancy of experimental points reduces at increasing of $\sqrt{s}$.

In the case of hadronic reaction influence of in the leading particles results in decreasing (increasing) of average value of $S(T)$ in comparison with $e^{+} e^{-}$and $l \mathrm{~N}$ collisions. Selection of events with high multiplicity, specific type of interaction (non-diffractive) or using of modified sphericity tensor allows to reduce the influence of the leading particles on jet characteristics in hadronic reactions. Additional cuts indicated above result in better agreement of values of $\langle S\rangle$ and $\langle T\rangle$ in various interactions in the range of intermediate energies. Experimental results for $\pi^{-} \mathrm{Ne}$ collisions at $6.2 \mathrm{GeV} / c[70,71]$ demonstrate the influence of nuclear matter on jet observables.
Exception of interactions with single intranuclear nucleon and, probably, small, in comparison with radius of a target nucleus, length of forming of final hadronic state [75] leads to essential influence of multiple rescattering on jet characteristics which results in wider jets and significantly larger (smaller) average values of sphericity (thrust) in comparison with results of other experiments at close energies. The account of interactions with a single intranuclear nucleon in case of discussed $\pi^{-} \mathrm{Ne}$ interactions leads to $\langle S\rangle=0.269 \pm 0.005$ and $\langle T\rangle=0.814 \pm 0.002$, that would be agreed with the general tendency much more reasonably. Reducing of influence of rescattering with increasing of beam momentum leads to improvement of agreement of $\langle S\rangle$ even for multinucleon hadron-nuclear interactions with the general tendency (Fig 2a), that is visible on an example of $\pi^{-} \mathrm{C}$ collisions [72]. Thus, integral (on number of participating nucleons) results confirm a conclusion $[71,72]$, that the mechanism of production of hadron jets at nuclei is more complex, than that in processes with hadrons and / or leptons, and growth of average values of sphericity for hA interactions at intermediate energies can be explained by multiple rescattering of secondary particles at nucleons of a target nucleus. Point for $\pi^{-} \mathrm{Ne}$ at initial momentum $6.2 \mathrm{GeV} / c$ discussed above is excluded below from the fitted samples for sphericity and thrust. At present there are no experimental results in TeV energy domain for traditional collective observables which were described above. The experimental results for transverse thrust $\left(T_{\perp}\right)$ from $[62,65]$ are used as estimations of thrust defined by (3) at $\mathrm{TeV}$ scale energies. This assumption is based on the relation $T \sim T_{\perp}$ which valids for events with two back-to-back jets and for near-to-planar 3-jet events $[76,77,78]$. As seen from Fig,2 the CDF experimental result [65] differs noticeably from general tendency for thrust 


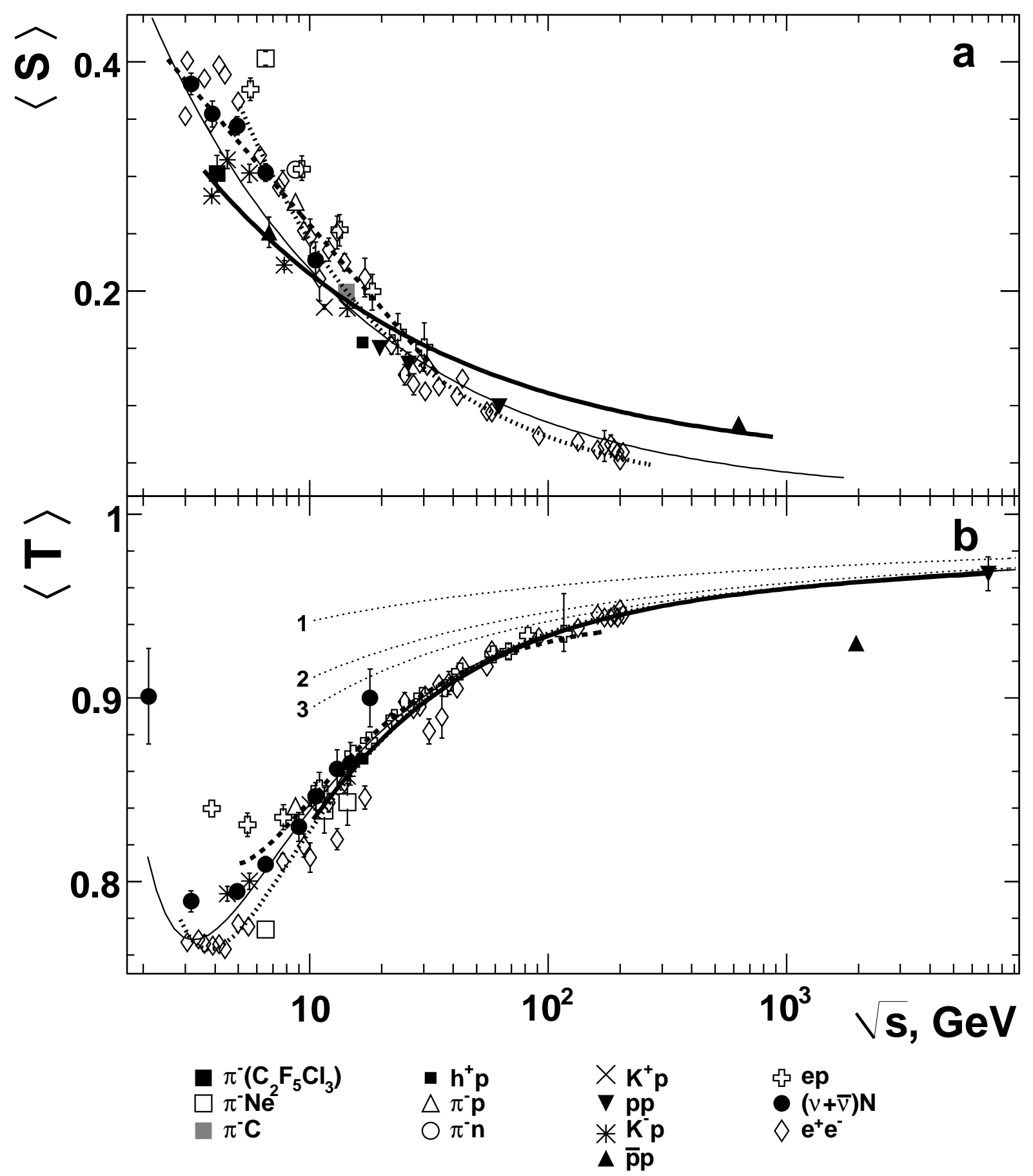

Fig. 2. Average values of the sphericity (a) and thrust (b) depend on initial energy. Experimental results and estimations are from database presented in Table 2. Total errors are shown. See text for explanation of the curves. 
Table 4. Results of fit of dependence $\langle S\rangle(s)$ by function (7)

\begin{tabular}{ccccc}
\hline Data sample & \multicolumn{4}{c}{ Fit parameters } \\
\cline { 2 - 5 }$a_{1}$ & $a_{2}$ & $a_{3}$ & $\chi^{2} / \mathrm{ndf}$ \\
\hline hh and $\mathrm{hA}$ & $0.047 \pm 0.004$ & $0.442 \pm 0.005$ & $-0.210 \pm 0.006$ & 97.5 \\
$l \mathrm{~N}$ & $-357 \pm 303$ & $358 \pm 303$ & $(-1.5 \pm 1.2) \times 10^{-4}$ & 5.22 \\
$e^{+} e^{-}(\sqrt{s}>5 \mathrm{GeV})$ & $0.019 \pm 0.004$ & $0.94 \pm 0.02$ & $-0.311 \pm 0.009$ & 2.95 \\
all data & $0.021 \pm 0.002$ & $0.610 \pm 0.004$ & $-0.244 \pm 0.004$ & 70.0 \\
\hline
\end{tabular}

observable 1 , i.e. the assumption is less correct for CDF than that for CMS. Possibly there are several reasons for such behavior of experimental point at $\sqrt{s}=1.96 \mathrm{TeV}$. As known contribution of soft particles at large angles with respect to the event plane can leads to $T \gtrsim T_{\perp}$ [77]. Indeed, CDF data show slightly intensive energy flow out of the hard-scattering plane [65]. Thus discrepancy of CDF point from general trend can be caused, in particular, by more significant contribution of soft particles at large angles. Additional reason may be kinematical cuts used in CDF analysis. In any case for verification of this hypothesis additional detail study is essential for event geometry with taking into account soft particles, especially, examination of relation $p_{\text {in }} \sim p_{\text {out }}$ for such particles. Therefore the CDF experimental point is excluded below from fitted samples.

There are smooth dependencies of experimental $\langle S\rangle$ on $\sqrt{s}$ for various interactions without qualitative changes at transition from intermediate energy domain to high energies (Fig 2a). Taking into account general view of sphericity dependence on initial energy and results for $e^{+} e^{-}[27$, $39]$, in the present paper the $\langle S\rangle(s)$ is approximated by power function

$$
\langle Y\rangle=a_{1}+a_{2} X^{a_{3}},
$$

where $Y$ is certain collective variable and $X$ denotes the appropriate argument of a function, $Y \equiv S$ and $X \equiv s$ in the case under considered. Fit results for various samples of experimental data for sphericity observable by function (77) are presented in Table 4 and shown on Fig 2 a for $\mathrm{hh}+\mathrm{hA}$ interactions by a solid line, for $l \mathrm{~N}-$ by dashed one, for $e^{+} e^{-}$annihilation - by dotted line and for the joint sample by thin solid one. The disorder of experimental points, especially in the range of $\sqrt{s} \leq 5-10 \mathrm{GeV}$, does not allow to obtain statistically acceptable fit quality. Influence of discrepancy of experimental data from indicated above energy domain on the approximation quality is confirmed, for example, by results for $e^{+} e^{-}$annihilation: for $\sqrt{s}>5 \mathrm{GeV}$ reasonable fit quality is observed (Table 4), moreover value of $a_{3}$ parameter agrees within errors with result from [27], on the other hand, fitting of all available energy range results in $\chi^{2} / \mathrm{ndf}=19.1$. However one can see from Fig 2, that the behavior of $\langle S\rangle$ depending on $\sqrt{s}$ is described by function (7) at a qualitative level both for

\footnotetext{
${ }^{1}$ One need to emphasize that the result based on partonlevel NLO+NLL distribution agree much better with general trend.
}

separate interactions, and for the joint sample for all available range of initial energies. Additional research for the joint sample with using of more complex fit function, in particular, the sum of power functions, has shown, that experimental data are better described by function (7).

Unlike sphericity, collective observable the thrust possesses infrared stability that allows to use QCD for study of behavior various dependencies and distributions on this collective parameter. In the framework of pQCD the energy dependence of the first moment of thrust distribution can be described by the following function

$$
\langle T\rangle^{\mathrm{pQCD}}(s)=1-\sum_{i=1} \overline{\mathcal{A}}_{i} \alpha_{S}^{i}(s), \quad \alpha_{S}(s)=\left(b_{0} t\right)^{-1} .
$$

Here $t \equiv \ln s / \Lambda^{2}, \Lambda$ is the QCD parameter, $b_{0}$ is referred to as one-loop $\beta$-function coefficient [2]. Parameters $\overline{\mathcal{A}}_{i}$ are calculated up to NNLO in [79] and for (8) they are $\overline{\mathcal{A}}_{1}=0.335, \overline{\mathcal{A}}_{2}=1.033, \overline{\mathcal{A}}_{3}=2.76 \pm 0.09$ for number of (light, i.e. active) quark flavors $N_{f}=5$. Fig $2 \mathrm{~b}$ shows the theoretical predictions for LO ("1"), NLO ("2") and NNLO ("3") pQCD based on the (8) by thin dotted curves. As seen behavior of a theoretical curve $\langle T\rangle^{\mathrm{pQCD}}(s)$ depends on the order of pQCD expansion, especially, in the intermediate energy domain. The agreement of theoretical prediction (8) with experimental data improves for higher order of pQCD expansion. The LO pQCD overpredicts the thrust value even at LHC energy. It is important to note very good agreement of experimental results for pp collisions at $\sqrt{s}=7 \mathrm{TeV}[62]$ and a theoretical curves (8) for NLO and NNLO. Such quantitative agreement is observed for the first time, especially, for hadronic reaction and allows to assume, that at $\sqrt{s} \sim 5-10 \mathrm{TeV}$ nonperturbative corrections become negligible. As known the contribution of these corrections increases at decreasing of initial energy and for approximation of $\langle T\rangle(s)$ in range of $\sqrt{s}$ as wide as possible it is necessary to use the phenomenological models which take into account the nonperturbative corrections. Thus, in the present work for fit of dependencies $\langle T\rangle(s)$ in various interactions the following general function is used:

$$
\langle T\rangle(s)=1-\left[\langle F\rangle^{\mathrm{pQCD}}+\langle F\rangle^{\mathrm{n}-\mathrm{p}}\right],
$$

where components $\langle F\rangle^{\mathrm{pQCD}}$ and $\langle F\rangle^{\mathrm{n}-\mathrm{p}}$ are model dependent. In the framework of present paper dispersive model (DM) $[80,81]$ and single dressed gluon model (SDGM) [82-84] have been used for approximation of dependence $\langle T\rangle(s)$. It is necessary to note, that now the most part 
of calculations are executed for electron-positron annihilation and comparison of models with experimental values of collective characteristics is carried out, as usual, for the this type of interactions because precision of measurements and calculations for shape variables of final hadronic state is the best for $e^{+} e^{-}$namely, in additional to significant sample of experimental results is available in the high energy domain where applicability of QCD and corresponding phenomenological models has more rigorous substantiation. Some predictions on the basis of DM have been obtained for $e p$ interactions also [49, 51, 52]. For hadron-hadron and hadron-nuclear reactions the number of phenomenological predictions is much less, that is connected both with the raised difficulty of calculations and with absence of experimental data at high energies until recently. Therefore the functional forms obtained for $e^{+} e^{-}$ initially are used below for fitting of various data samples.

In DM perturbative part is defined in the framework of NLO pQCD and terms for (9) are the following:

$$
\begin{aligned}
& \langle F\rangle_{\mathrm{DM}}^{\mathrm{pQCD}}=\sum_{i=1}^{2} \mathcal{A}_{i} \bar{\alpha}_{S}^{i}(s), \quad \bar{\alpha}_{S}(s) \equiv \alpha_{S}(s) / 2 \pi, \\
& \langle F\rangle_{\mathrm{DM}}^{\mathrm{n}-\mathrm{p}}=\mathcal{M} \frac{4 C_{F}}{\pi}\left(\frac{\mu_{I}}{\sqrt{s}}\right) \times \\
& {\left[\bar{\alpha}_{0}\left(\mu_{I}\right)-\alpha_{S}(s)-b_{0}\left(\ln \frac{s}{\mu_{I}^{2}}+\frac{K}{2 \pi b_{0}}+2\right) \alpha_{S}^{2}(s)\right],} \\
& K=\left(\frac{67}{18}-\frac{\pi^{2}}{6}\right) C_{A}-\frac{5}{9} N_{f}, C_{F}=\frac{4}{3}, C_{A}=3 .
\end{aligned}
$$

Here $\mathcal{M}$ is correcting so called Milan factor [85, 86]. In this work $\mathcal{M}$ and $\mathcal{A}_{i},(i=1,2)$ are free fit parameters. Non-perturbative parameter

$$
\bar{\alpha}_{p}\left(\mu_{I}\right)=\frac{p+1}{\mu_{I}^{p+1}} \int_{0}^{\mu_{I}} k^{p} \alpha_{S}(k) d k
$$

is introduced for the account of a part of expression for $\alpha_{S}(s)$ which diverges below $\mu_{I}$ - infrared matching scale [80]. There is no unambiguous scheme for definition of $\mu_{I}$ value. The empirical choice of $\mu_{I}$ and $N_{f}$ values is restricted by the requirement of validity of following conditions: $\Lambda \ll \mu_{I}<\sqrt{s_{\min }}$ [80] and $\forall N_{f}: m_{q}<\sqrt{s_{\min }}[2]$, where $\sqrt{s_{\text {min }}}$ is the low boundary of fitted energy range, $m_{q}$ denotes masses of the quarks, which can be considered as lights for a certain $\sqrt{s_{\min }}$. One need to emphasize that in the framework of present paper, in distinction from, for example [51], for $l \mathrm{~N}$ collisions dependencies of parameters $\mathcal{A}_{i}, \quad(i=1,2)$ on some kinematic quantities $(x, Q)$ have not been taken into account because, as indicated above, the averaged on these parameters and various experiments values of thrust are used. Fit results for various samples of experimental data for thrust observable by function (9) in the framework of DM are presented in Table 5 and shown on Fig $2 \mathrm{~b}$ for $e^{+} e^{-}$annihilation by dotted curve, for $l \mathrm{~N}-$ by dashed one, for hh $+\mathrm{hA}$ interactions - by solid line, and for the joint sample - by thin solid one. As usual the better fit quality is obtained at the fixed value of $\mathcal{M}$ which is defined by limits of the allowable interval of Milan factor values [85]. As seen from Table 5 and Fig $2 \mathrm{~b}$ DM allows to describe all available results for $e^{+} e^{-}$at reasonable fit quality. The disorder of experimental points leads to that statistically acceptable values of $\chi^{2} /$ ndf are observed at $\sqrt{s_{\min }}=5$ and $10 \mathrm{GeV}$ only for $l \mathrm{~N}$ and hadronic reactions respectively. For these interactions the fit functions are shown Fig $2 b$ at $\sqrt{s_{\min }}$ indicated above namely. However function (9) in the framework of DM allows to describe dependence $\langle T\rangle(s)$ at a qualitative level both for some interactions and for the joint data sample in all range of available initial energies (Fig,2b). Behavior of DM-curves agrees very well with NLO and NNLO pQCD predictions in the LHC energy domain. Thus, for the first time the reasonable phenomenological description is obtained for experimental results for thrust variable in all available energy range both for $e^{+} e^{-}$and for the joint data sample for various interactions. Non-perturbative parameter $\bar{\alpha}_{p}\left(\mu_{I}\right)$ values obtained for $e^{+} e^{-}$and $l \mathrm{~N}$ at $\sqrt{s_{\min }}=10 \mathrm{GeV}$ agree with previous results $[52,87]$ within errors. Clear dependence of $\bar{\alpha}_{p}\left(\mu_{I}\right)$ on $\sqrt{s_{\min }}$ is absent within errors for these interactions. The similar situation is observed for hadronic reactions. But one can see growth of $\bar{\alpha}_{p}\left(\mu_{I}\right)$ value at decreasing of $\sqrt{s_{\min }}$ for joint data sample, especially visible at transition in strong non-perturbative region from $\sqrt{s_{\min }}=5 \mathrm{GeV}$ down to $\sqrt{s_{\min }}=2 \mathrm{GeV}$ (Table 5). There is no clear dependence of $\bar{\alpha}_{p}\left(\mu_{I}\right)$ value on type of interaction at fixed low boundary for fitted energy domain.

For $e^{+} e^{-}$results and for joint data sample approximations in the framework of SDG-model have been investigated too. In the case of the first moment of thrust distribution, $\langle T\rangle$, perturbative and non-perturbative components in SDGM for (9) are the following [87]:

$$
\langle F\rangle_{\mathrm{SDGM}}^{\mathrm{pQCD}}=\sum_{i=1}^{6} \mathcal{B}_{i} \bar{a}^{i}(s) / \pi,\langle F\rangle_{\mathrm{SDGM}}^{\mathrm{n}-\mathrm{p}}=\nu / \sqrt{s} .
$$

Here

$$
\bar{a}(s) \equiv \frac{\alpha_{S}(s)}{1-\frac{5}{3} b_{0} \alpha_{S}(s)}
$$

and $\nu$ is a free fit parameter. Coefficients $\mathcal{B}_{i}$ are defined via so called $\log$ moments $d_{i}$ of the characteristic thrust function, calculated in [83], by following way

$$
\begin{aligned}
\mathcal{B}_{1}= & d_{0}, \mathcal{B}_{2}=\beta_{0} d_{1}, \mathcal{B}_{3}=\left(-\frac{d_{0} \pi^{2}}{3}+d_{2}\right) \beta_{0}^{2}+\beta_{1} d_{1} \\
\mathcal{B}_{4}= & \left(-\pi^{2} d_{1}+d_{3}\right) \beta_{0}^{3}+\frac{5}{2} \beta_{1} \beta_{0} d_{2}+\beta_{2} d_{1}, \\
\mathcal{B}_{5}= & \left(d_{4}+\frac{d_{0} \pi^{4}}{5}-2 \pi^{2} d_{2}\right) \beta_{0}^{4}+\left(\frac{13}{3} \beta_{1} d_{3}-\beta_{1} \pi^{2} d_{1}\right) \beta_{0}^{2} \\
& +3 \beta_{2} d_{2} \beta_{0}+\frac{3}{2} \beta_{1}^{2} d_{2}+\beta_{3} d_{1}, \\
\mathcal{B}_{6}= & \left(-\frac{10}{3} \pi^{2} d_{3}+\pi^{4} d_{1}+d_{5}\right) \beta_{0}^{5}+\left(\frac{77}{12} d_{4}-\frac{9}{2} \pi^{2} d_{2}\right) \times \\
& \beta_{1} \beta_{0}^{3}+\left(6 d_{3}-\pi^{2} d_{1}\right) \beta_{2} \beta_{0}^{2}+\left(\frac{35}{6} \beta_{1}^{2} d_{3}+\frac{7}{2} \beta_{3} d_{2}\right) \beta_{0}+ \\
& \beta_{4} d_{1}+\frac{7}{2} \beta_{1} \beta_{2} d_{2} .
\end{aligned}
$$


Table 5. Results for fit of dependence $\langle T\rangle(s)$ by function (9) in the framework of DM

\begin{tabular}{|c|c|c|c|c|}
\hline \multirow{2}{*}{$\begin{array}{c}\text { Fit } \\
\text { parameter }\end{array}$} & \multicolumn{4}{|c|}{ Data sample } \\
\hline & $e^{+} e^{-}$ & $l \mathrm{~N}$ & hh and $\mathrm{hA}$ & all data \\
\hline \multicolumn{5}{|c|}{$\sqrt{s_{\min }}=2 \mathrm{GeV}\left(\mu_{I}=1 \mathrm{GeV}, N_{f}=3\right)$} \\
\hline $\mathcal{A}_{1}$ & $3.8 \pm 0.4$ & $2.1 \pm 0.3$ & $4.08 \pm 0.15$ & $2.6 \pm 0.3$ \\
\hline $\mathcal{A}_{2}$ & $-63 \pm 26$ & $48 \pm 17$ & $-11.1 \pm 0.8$ & $16 \pm 6$ \\
\hline $\mathcal{M}$ (fixed) & 1.795 & 1.448 & 1.100 & 1.100 \\
\hline $\bar{\alpha}_{0}\left(\mu_{I}\right)$ & $0.77 \pm 0.04$ & $0.57 \pm 0.03$ & $0.628 \pm 0.012$ & $0.75 \pm 0.05$ \\
\hline$\alpha_{S}\left(M_{Z}\right)$ & $0.1192 \pm 0.0007$ & $0.1209 \pm 0.0012$ & $0.1180 \pm 0.0011$ & $0.1218 \pm 0.0007$ \\
\hline$\chi^{2} / \mathrm{ndf}$ & 3.04 & 8.16 & 20.1 & 15.6 \\
\hline \multicolumn{5}{|c|}{$\sqrt{s_{\min }}=5 \mathrm{GeV}\left(\mu_{I}=2 \mathrm{GeV}, N_{f}=4\right)$} \\
\hline $\mathcal{A}_{1}$ & $3.17 \pm 0.13$ & $8.02 \pm 0.06$ & $4.2 \pm 1.2$ & $2.76 \pm 0.15$ \\
\hline $\mathcal{A}_{2}$ & $-32 \pm 4$ & $-300 \pm 7$ & $6 \pm 3$ & $2.7 \pm 1.3$ \\
\hline $\mathcal{M}$ (fixed) & 1.520 & 1.852 & 1.188 & 1.188 \\
\hline $\bar{\alpha}_{0}\left(\mu_{I}\right)$ & $0.532 \pm 0.018$ & $0.638 \pm 0.008$ & $0.39 \pm 0.02$ & $0.517 \pm 0.008$ \\
\hline$\alpha_{S}\left(M_{Z}\right)$ & $0.1203 \pm 0.0018$ & $0.1222 \pm 0.0003$ & $0.116 \pm 0.004$ & $0.1206 \pm 0.0009$ \\
\hline$\chi^{2} / \mathrm{ndf}$ & 3.63 & 1.74 & 20.3 & 7.38 \\
\hline \multicolumn{5}{|c|}{$\sqrt{s_{\min }}=10 \mathrm{GeV}\left(\mu_{I}=2 \mathrm{GeV}, N_{f}=5\right)$} \\
\hline $\mathcal{A}_{1}$ & $4.4 \pm 1.3$ & $-0.6 \pm 0.3$ & $2.7 \pm 1.1$ & $3.7 \pm 1.2$ \\
\hline $\mathcal{A}_{2}$ & $-97 \pm 66$ & $212 \pm 21$ & $8 \pm 2$ & $-58 \pm 34$ \\
\hline $\mathcal{M}$ & 1.291 (fixed) & 1.291 (fixed) & 1.920 (fixed) & $1.8 \pm 0.5$ \\
\hline $\bar{\alpha}_{0}\left(\mu_{I}\right)$ & $0.59 \pm 0.18$ & $0.32 \pm 0.17$ & $0.42 \pm 0.10$ & $0.49 \pm 0.04$ \\
\hline$\alpha_{S}\left(M_{Z}\right)$ & $0.115 \pm 0.011$ & $0.115 \pm 0.017$ & $0.117 \pm 0.016$ & $0.121 \pm 0.011$ \\
\hline$\chi^{2} / \mathrm{ndf}$ & 3.28 & 0.43 & 0.69 & 3.19 \\
\hline
\end{tabular}

Table 6. Results for fit of dependence $\langle T\rangle(s)$ by function (9) in the framework of SDGM

\begin{tabular}{cccc}
\hline Data & \multicolumn{3}{c}{ Fit parameter } \\
\cline { 2 - 4 } sample & $\alpha_{S}\left(M_{Z}\right)$ & $\nu$ & $\chi^{2} /$ ndf \\
\hline$e^{+} e^{-}$ & $0.1235 \pm 0.0008$ & $-0.28 \pm 0.09$ & 6.98 \\
all data & $0.1197 \pm 0.0005$ & $-0.142 \pm 0.049$ & 14.0 \\
\hline
\end{tabular}

Here $\beta_{j}=(4 \pi)^{j+1} b_{j}, j=0-4, b_{j}$ are coefficients of $\beta$-functions in $j$-loop approach [2] with that $\beta_{4}=0$ because of $b_{4}$ is unknown [87]. Scheme described above for DM is used in this case too for choice of $N_{f}$ depending on $\sqrt{s_{\min }}$ value. Detail study shown, that at the fixed values of $d_{i}$ from [83] SDGM allows to obtain the description of experimental data at a qualitative level for both considered samples only at $\sqrt{s_{\min }}=10 \mathrm{GeV}$ (Table 6) with that presence of non-perturbative term weakly influences at fit quality. Expansion of fitted range down to the lower energies leads to significant discrepancy of fit curve with experimental data.

Fig 3 shows values of $\alpha_{S}\left(M_{Z}\right)$ obtained by fit of dependence $\langle T\rangle(s)$ in the framework of DM and SDGM at various $\sqrt{s_{\text {min }}}$. Experimental values of $\alpha_{S}\left(M_{Z}\right)$ derived at $\sqrt{s_{\min }}=2$ and $5 \mathrm{GeV}$ for some interactions as well as for joint data sample agree reasonably with world average $[2,88]$. Precision for estimations of strong coupling constant considerably deteriorates at $\sqrt{s_{\min }}=10 \mathrm{GeV}$ in comparison with smaller values of the low boundary of fitted energy range in the framework of DM (Fig, 3 ). At this value of $\sqrt{s_{\min }} \mathrm{SDGM}$ allows to improve significantly the precision of $\alpha_{S}\left(M_{Z}\right)$ estimation. The value of $\alpha_{S}\left(M_{Z}\right)$ extracted in the framework of SDGM for the joint sample is in good agreement with world average value $\alpha_{S}^{\text {wa }}\left(M_{Z}\right)$. On the other hand there is some excess of experimental value of strong coupling constant for $e^{+} e^{-}$sample with respect to the $\alpha_{S}^{\text {wa }}\left(M_{Z}\right)$. However $\alpha_{S}\left(M_{Z}\right)$ experimental value shown in Table 6 for $e^{+} e^{-}$coincides within errors with the results obtained, for example, at investigation of radiative decays of heavy quarkonium $(\Upsilon)$ and shape of final hadronic state in ALEPH experiment [2]. Thus, investigation of energy dependence of average thrust values for various interactions allows to extract estimations of $\alpha_{S}\left(M_{Z}\right)$ which agree reasonably both with world average and with results of other methods.

Figure 4 shows energy dependence for $\left\langle p_{\text {in }}^{2}\right\rangle$ (a), $\left\langle p_{\text {out }}^{2}\right\rangle$ (b), $\langle\mathrm{A}\rangle(\mathrm{c})$ and $\langle\mathrm{P}\rangle(\mathrm{d})$. Experimental results and the estimations for collective observables are taken from a database presented in Table2, Dependencies for all collective parameters under study show smooth behavior, without qualitative changes at transition from intermediate 


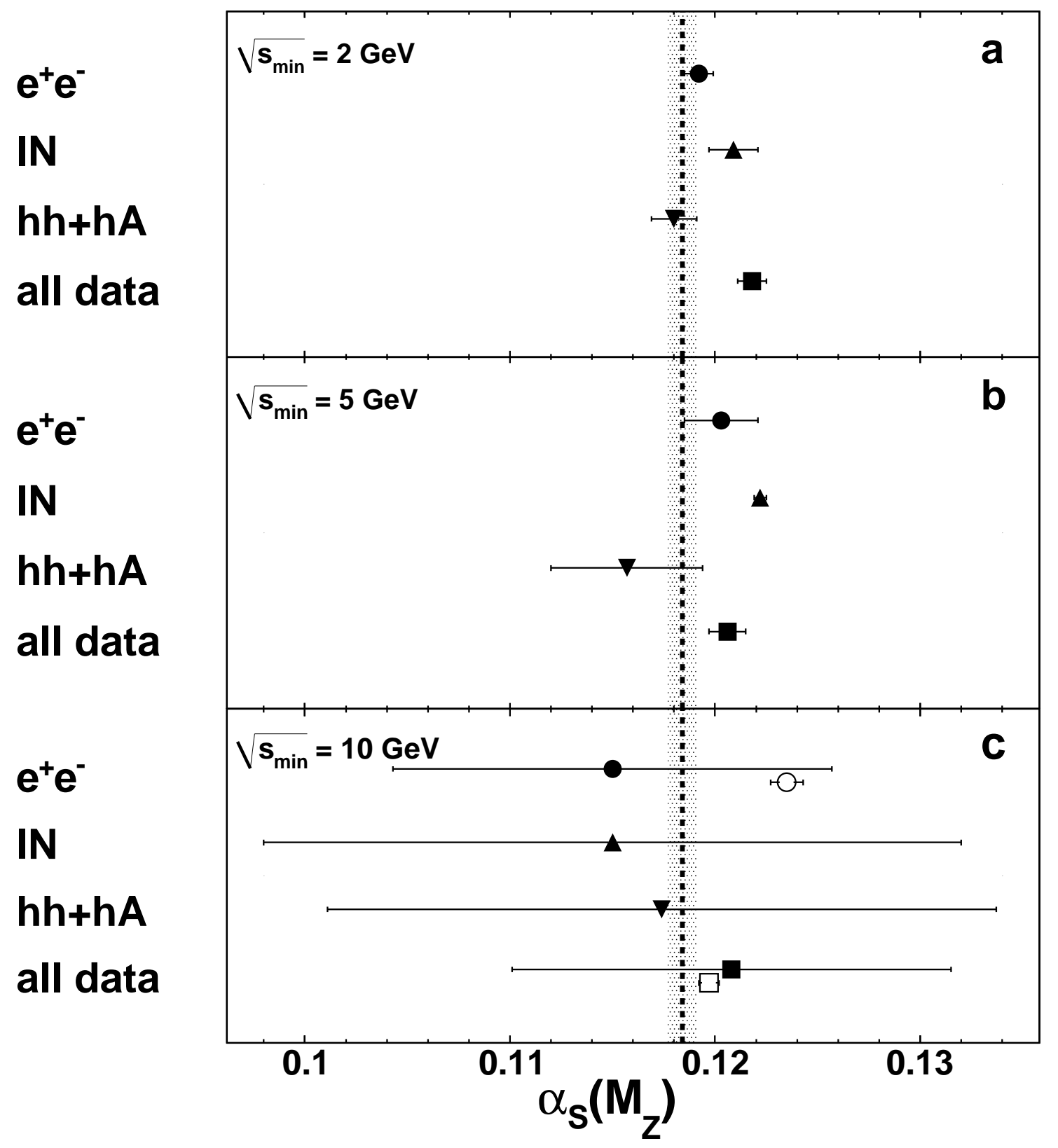

Fig. 3. Values of $\alpha_{S}\left(M_{Z}\right)$ obtained by fitting of various samples of experimental data at $\sqrt{s_{\min }}=2$ (a), 5 (b) and $10 \mathrm{GeV}$ (c). Values extracted in the framework of DM are shown by closed symbols, for SDGM - by opened ones. Dashed line and shared band are shown the world average value with errors $\alpha_{S}^{\text {wa }}\left(M_{Z}\right)=0.1184 \pm 0.0007[2,88]$.

energy domain to high energies (Fig,4). Unlike the situation for one of the main collective parameters, $T$, the amount of phenomenological models describing behavior of observables depending on initial energy considered on Fig 4, is essential less or they are absent at all. Therefore in the framework of present paper the wide set of functions has been studied for empirical approximations of experimental results for total available energy range.

Experimental results for hadron-nuclear $\pi^{-}$Ne interactions agree both with results for hadron-hadron reactions at similar energies and with general trends reasonably in case of a component of a transverse momentum in "principal axes" frame (Fig 4 and Fig $4 \mathrm{~b}$ ). On the other hand the multinucleon $\pi^{-}$Ne collisions show essentially larger values of parameters $\langle\mathrm{A}\rangle$ (Fig 4 $)$ and $\langle\mathrm{P}\rangle$ (Fig $4 \mathrm{~d})$, i.e. hadron-nuclear collisions at $\sqrt{s} \simeq 6 \mathrm{GeV}$ are characterized by weaker alignment of hadrons with respect to the jet axis and smaller planarity of events in comparison with hadronic interactions, that can be explained, possibly, by rescattering of hadronic states in nuclear matter discussed above. Therefore the experimental results for $\pi^{-}$Ne are ex- 

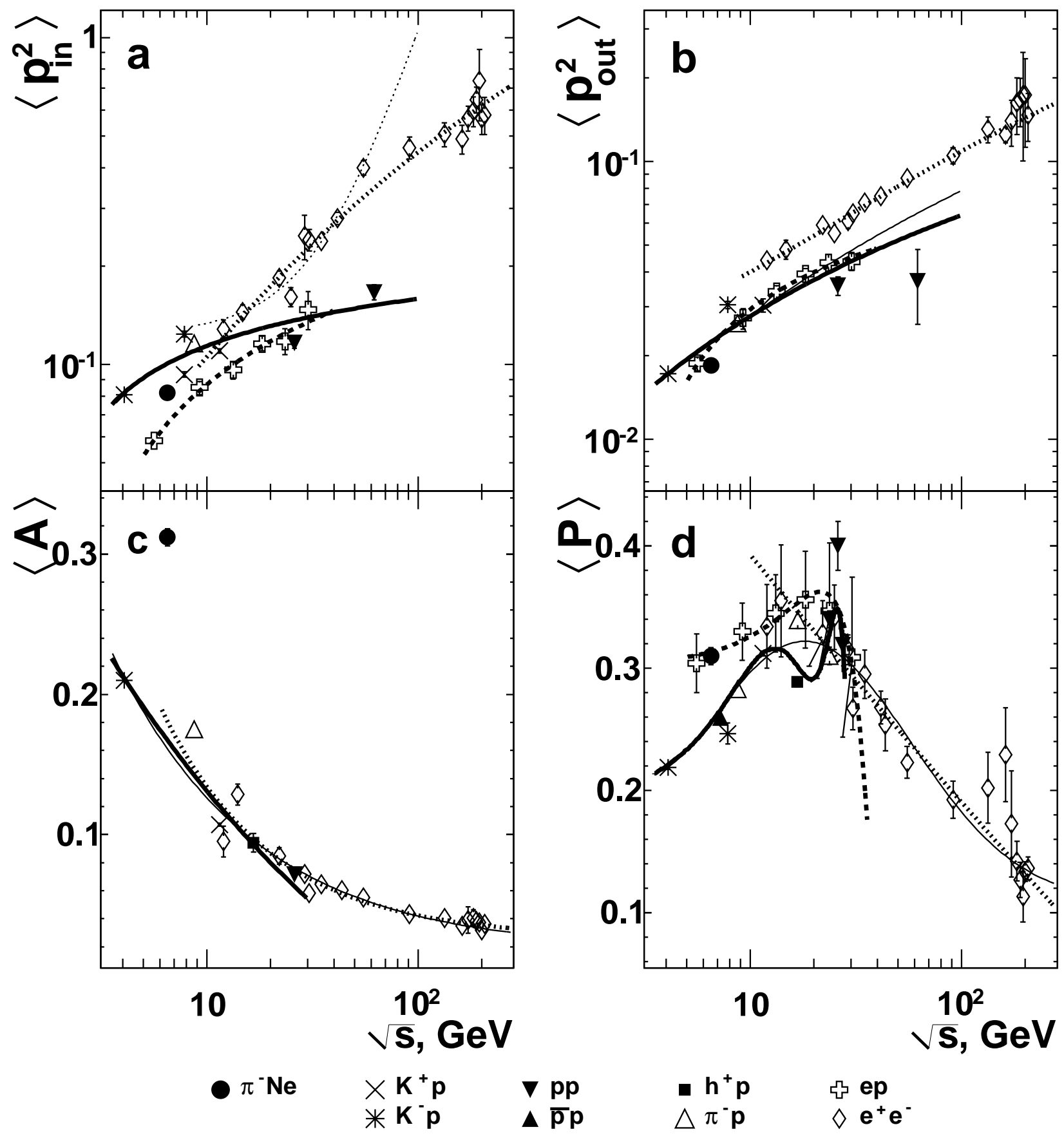

Fig. 4. Average values of the parameters describing the event shape in the "principal axes" coordinate system depend on initial energy: $\mathrm{a}-\left\langle p_{\text {in }}^{2}\right\rangle, \mathrm{b}-\left\langle p_{\text {out }}^{2}\right\rangle, \mathrm{c}-\langle\mathrm{A}\rangle, \mathrm{d}-\langle\mathrm{P}\rangle$. Values of $\left\langle p_{\text {in }}^{2}\right\rangle$ and $\left\langle p_{\text {out }}^{2}\right\rangle$ are shown in $(\mathrm{GeV} / c)^{2}$. Experimental results and estimations are from database presented in Table 2 Total errors are shown. See text for explanation of the curves. 
Table 7. Fit results for energy dependence of collective observables under considered

\begin{tabular}{|c|c|c|c|c|c|}
\hline \multirow{2}{*}{$\begin{array}{c}\text { Data } \\
\text { sample } \\
\end{array}$} & \multirow[t]{2}{*}{ Function } & \multicolumn{4}{|c|}{ Fit parameter } \\
\hline & & $a_{1}$ & $a_{2}$ & $a_{3}$ & $\chi^{2} / \mathrm{ndf}$ \\
\hline \multicolumn{6}{|c|}{$\left\langle p_{\text {in }}^{2}\right\rangle$} \\
\hline $\begin{array}{c}\text { hh and hA } \\
e \mathrm{p} \\
e^{+} e^{-} \\
\end{array}$ & $\begin{array}{l}(12) \\
-/ /- \\
-/ /-\end{array}$ & $\begin{array}{c}-36 \pm 28 \\
-0.08 \pm 0.03 \\
0.033 \pm 0.025 \\
\end{array}$ & $\begin{array}{c}36 \pm 28 \\
0.06 \pm 0.02 \\
(1.6 \pm 0.8) \times 10^{-3} \\
\end{array}$ & $\begin{array}{c}(1.8 \pm 1.4) \times 10^{-3} \\
0.7 \pm 0.3 \\
2.5 \pm 0.3 \\
\end{array}$ & $\begin{array}{l}51 \\
0.53 \\
2.24 \\
\end{array}$ \\
\hline \multicolumn{6}{|c|}{$\left\langle p_{\text {out }}^{2}\right\rangle$} \\
\hline $\begin{array}{c}\text { hh and hA } \\
e p \\
(\mathrm{hh}, \mathrm{hA})+e \mathrm{p} \\
e^{+} e^{-} \\
\end{array}$ & $\begin{array}{l}(12) \\
-/ /- \\
-/ /- \\
-/ /-\end{array}$ & $\begin{array}{c}0.007 \pm 0.003 \\
-0.119 \pm 0.009 \\
(1.08 \pm 0.02) \times 10^{-2} \\
0.024 \pm 0.007 \\
\end{array}$ & $\begin{array}{c}(2.1 \pm 0.8) \times 10^{-3} \\
0.099 \pm 0.007 \\
(1.6 \pm 0.04) \times 10^{-3} \\
(4 \pm 2) \times 10^{-4} \\
\end{array}$ & $\begin{array}{c}1.5 \pm 0.3 \\
0.26 \pm 0.02 \\
1.0 \text { (fixed) } \\
2.4 \pm 0.3 \\
\end{array}$ & $\begin{array}{c}31 \\
0.19 \\
12 \\
0.79 \\
\end{array}$ \\
\hline \multicolumn{6}{|c|}{$\langle\mathrm{A}\rangle$} \\
\hline $\begin{array}{c}\text { hh } \\
e^{+} e^{-} \\
\text {all data }\end{array}$ & $\begin{array}{l}(7) \\
-/ /- \\
-/ /-\end{array}$ & $\begin{array}{l}-0.19 \pm 0.07 \\
0.027 \pm 0.004 \\
0.017 \pm 0.002 \\
\end{array}$ & $\begin{aligned} & 0.57 \pm 0.06 \\
& 0.7 \pm 0.2 \\
& 0.483 \pm 0.008 \\
&\end{aligned}$ & $\begin{array}{c}-0.12 \pm 0.03 \\
-0.42 \pm 0.06 \\
-0.323 \pm 0.008 \\
\end{array}$ & $\begin{array}{l}195 \\
2.29 \\
25.3 \\
\end{array}$ \\
\hline \multicolumn{6}{|c|}{$\langle\mathrm{P}\rangle$} \\
\hline $\begin{array}{c}e \mathrm{p} \\
e^{+} e^{-}\end{array}$ & $\frac{(13)}{(7)}$ & $\begin{array}{c}0.30 \pm 0.02 \\
-3.24 \pm 0.07\end{array}$ & $\begin{array}{c}(2.6 \pm 1.4) \times 10^{-4} \\
3.85 \pm 0.07\end{array}$ & $\begin{array}{c}(-2.8 \pm 1.7) \times 10^{-7} \\
\quad-0.012 \pm 0.001\end{array}$ & $\begin{array}{c}0.16 \\
0.88\end{array}$ \\
\hline
\end{tabular}

cluded from the fit samples in the case of $\langle\mathrm{A}\rangle$ (Fig $4 \mathrm{c})$ and $\langle\mathrm{P}\rangle($ Fig $4 \mathrm{~d})$ variables below.

Comparison of Fig 4 a with Fig $4 \mathrm{~b}$ demonstrates that value of "in"-component of transverse momentum is larger than corresponding "out"-component value at all considered initial energy values $\sqrt{s} \geq 4 \mathrm{GeV}$ and for all interaction types. In the energy domain accessible for comparison, values of $\left\langle p_{\text {in }}^{2}\right\rangle$ (Fig $4 \mathrm{a}$ ) and $\left\langle p_{\text {out }}^{2}\right\rangle$ (Fig $4 \mathrm{~b}$ ) show faster growth at increasing of $\sqrt{s}$ in case of $e^{+} e^{-}$annihilation in comparison with interactions of other types. It is agreed with results of the comparative analysis executed in [60] for mean square of transverse momentum, $\left\langle p_{\perp}^{2}\right\rangle$, at $10<\sqrt{s}<35 \mathrm{GeV}$. In the framework of present paper energy dependence for first moments of distributions of "in"- and "out"-component of transverse momentum is fitted by function

$$
\left\langle p_{j}^{2}\right\rangle(s)=a_{1}+a_{2}[\ln s]^{a_{3}}, \quad j \equiv \text { in, out. }
$$

Here $s$ is in $\mathrm{GeV}^{2}$. Results of fit of components of transverse momentum by function (12) are presented in Table 7 for various experimental data samples and shown on Fig 4 a,b for hh and hA collisions by a solid line, for $e p$ - by dashed line and for $e^{+} e^{-}$annihilation - by dotted one. The behavior of $\left\langle p_{\text {in }}^{2}\right\rangle(s)$ and $\left\langle p_{\text {out }}^{2}\right\rangle(s)$ depends on type of interaction, especially visible for the first observable. Disorder of experimental points and small errors do not allow to get reasonable fit quality for hadronic interactions, however function (12) agrees with the general tendency of experimental results at a qualitative level. Usually experimental data and estimations for $e^{+} e^{-}$and $e p$ are described by the offered function (12) with statistically acceptable quality both for $\left\langle p_{\text {in }}^{2}\right\rangle$ (Fig 4 a ), and for $\left\langle p_{\text {out }}^{2}\right\rangle$ (Fig 4b). As it has been stressed above, the estimations derived on the basis of published $e^{+} e^{-}$experimental data are used for, in particular, $\left\langle p_{\text {in }}^{2}\right\rangle$ and $\left\langle p_{\text {out }}^{2}\right\rangle$ at $\sqrt{s}>M_{Z}$. Direct experimental measurements are available for these observables up to $\sqrt{s}=55.2 \mathrm{GeV}$ in $e^{+} e^{-}$annihilation [21]. Therefore results for $e^{+} e^{-}$have been studied in energy range $\sqrt{s}<60 \mathrm{GeV}$ separately, in which $\left\langle p_{\text {in }}^{2}\right\rangle$ and $\left\langle p_{\text {out }}^{2}\right\rangle$ obtained from experiment data directly. For parameter $\left\langle p_{\text {in }}^{2}\right\rangle$ better fit quality turns out for power function (7) at $Y \equiv p_{\text {in }}^{2}$ in case under considered. Fit function for energy range indicated above is shown on Fig 4 a by thin dotted line, values of fit parameters are following: $a_{1}=0.124 \pm 0.005, a_{2}=(9.3 \pm 0.6) \times 10^{-5}, a_{2}=$ 1.0 (fixed), $\chi^{2} / \mathrm{ndf}=1.62$. Thus, measurements only at $\sqrt{s}<60 \mathrm{GeV}$ assume significantly faster growth of $\left\langle p_{\text {in }}^{2}\right\rangle$, than that observed at the account of estimations at LEP energies. For $\left\langle p_{\text {out }}^{2}\right\rangle$ the published results and estimations at collision energies larger than $M_{Z}$ show very smooth behavior without any features. Values of fit parameter for (12) are coincide within errors for fitting of all energy range and for $\sqrt{s}<60 \mathrm{GeV}$ domain only. Fitting by function (12) has been made for joint sample of results for hadronic and ep interactions because of close values of $\left\langle p_{\text {out }}^{2}\right\rangle$ for these collisions. The values of fit parameters presented in Table 7, close within errors with results for hh + hA data sample.

Alignment of secondary particles with respect to the jet axis amplifies with increasing of collision energy both in hadronic and in $e^{+} e^{-}$interactions (Fig 4 c). In the framework of present paper dependence $\langle\mathrm{A}\rangle(s)$ is fitted by function (7), where $Y \equiv \mathrm{A}$ in case under study. Fit results for various samples of experimental data are presented Table 7] and shown on Fig 4 4 by solid line for hh interactions, for $e^{+} e^{-}$annihilation - by dotted one and for sample of all 
available data - by thin solid curve. As seen from Fig 4 the behavior of collective observable $\langle\mathrm{A}\rangle$ depending on initial energy is described by function (7) for all sample under study at a qualitative level.

Absence of experimental data in the range of high / low energies for (hh, ep) / $e^{+} e^{-}$interactions respectively allows to propose only qualitative hypothesis concerning of behavior of planarity parameter depending on initial energy. Observable growth of $\langle\mathrm{P}\rangle$ up to $\sqrt{s} \sim 30 \mathrm{GeV}$ evidences on broadening jets in a transverse plane, that, probably, is dominated by the contribution of soft particles and more isotropic distributions of particle momenta in a transverse plane. At increasing of initial energy events become more and more planar (Fig 4 $\mathrm{d})$. In the present work dependence of planarity parameter on collision energy for $e p$ interactions is approximated by a polynomial function

$$
\langle\mathrm{P}\rangle=\sum_{k=0}^{N} a_{k} s^{k}
$$

of 2-d order and by function (7) - for $e^{+} e^{-}$annihilation. Results for fitting of these experimental data samples are presented in Table 7 and shown on Fig $4 \mathrm{~d}$ by dashed curve for $e \mathrm{p}$ interactions and by dotted line for $e^{+} e^{-}$annihilation. As fitting function of sample of experimental data for hh interactions had been considered power function (7), predicting smooth growth of planarity in collisions of the specified type at high energies, and polynomial function (13) of various orders (in the range of $N=2-8$ ). Better quality of approximation among the studied functions is observed for function (13) at $N=5\left(\chi^{2} / \mathrm{ndf}=11.9\right)$ for which following values of parameters have been obtained: $a_{1}=0.192 \pm 0.002, a_{2}=(1.84 \pm 0.07) \times 10^{-3}, a_{3}=$ $(-9.1 \pm 0.5) \times 10^{-6}, a_{4}=(1.71 \pm 0.11) \times 10^{-8}, a_{5}=$ $(-1.05 \pm 0.08) \times 10^{-11}$. This fit by function (13) for hh interaction sample shown on Fig $4 \mathrm{~d}$ by solid line, demonstrate fast decreasing of $\langle\mathrm{P}\rangle$ at $\sqrt{s}>30 \mathrm{GeV}$. The reason of such behavior, as well as in case of ep collisions, possibly, is absence of experimental data at $\sqrt{s}>30 \mathrm{GeV}$. Thus, additional experimental data are necessary for the quantitative analysis and more unambiguous conclusion concerning the behavior of $\langle\mathrm{P}\rangle(s)$ in hadronic interactions at high energies. During investigation of $\langle\mathrm{P}\rangle$ for all domain $\sqrt{s}>2 \mathrm{GeV}$ different versions of additional term in (7) have been examined, in particular, Landau, normal and log normal functions. These researches have shown, that joint sample of experimental results for hh, ep and $e^{+} e^{-}$ collisions supposes fit by the function

$$
\langle\mathrm{P}\rangle=a_{1}+a_{2} s^{a_{3}}+\frac{a_{4}}{s} \exp \left[-\frac{1}{2}\left(\frac{\ln s-a_{5}}{a_{6}}\right)^{2}\right]
$$

with the best $\chi^{2} / \mathrm{ndf}=5.36$ among studied functions and with the following values of fit parameters: $a_{1}=$ $0.11 \pm 0.02, a_{2}=2.3 \pm 1.3, a_{3}=-1.8 \pm 0.9, a_{4}=$ $-1043 \pm 587, a_{5}=11.2 \pm 0.9, a_{6}=2.3 \pm 0.2$. As seen from Fig 4d the suggested approximation function shown by thin solid line agrees qualitatively with experimental results at intermediate energies and corresponds to expected amplification of event planarity in high energy domain.

On the basis of the above experimental results, estimations and the suggested approximations it is possible to draw an intermediate conclusion, that all traditional collective variables show smooth dependence on energy without any features at $\sqrt{s} \sim 3-5 \mathrm{GeV}$. In Table 8 estimations are shown for parameters of event geometry in "principal axes" coordinate system in various interactions for the present and possible future accelerating facilities, calculated in the framework of the paper on the basis of analytical functions (7), (9) - (11), (12) - (14) and Table 4-7. Additional experimental results are essential for improving of precision of estimations for $\left\langle p_{\text {in }}^{2}\right\rangle$ and $\left\langle p_{\text {out }}^{2}\right\rangle$. But it would be emphasize, that the estimation of absolute value of transverse particle momentum with respect to the jet axis $\sqrt{\left\langle p_{\perp}^{2}\right\rangle}=0.50 \pm 0.17 \mathrm{GeV} / c$ derived in the framework of present paper for pp collisions at $\sqrt{s}=200$ $\mathrm{GeV}$ coincides within errors with experimental result at the same energy [89]. Study the high and ultra high energy domain show that the asymptotic values within errors are predicted for most collective parameters under study at $\sqrt{s} \sim 5-7 \mathrm{TeV}$ already.

\section{Multiplicity dependence}

Unlike energy dependence, the investigations of geometry of a hadronic final state at the fixed secondary particle multiplicities are less extensive and have been made for energy range $\sqrt{s}<60 \mathrm{GeV}$. Hadron-hadron interactions were studied in the exclusive approach in the some cases for intermediate energies. Results for collective observables were obtained only for jets of all identified secondary particles in some experiments (see, for example, $[22])$. Moreover using of total multiplicity $\left(N_{\text {tot }}\right)$ allows to increase amount of experimental points and hence to investigate behavior of dependence of jet characteristics on multiplicity in more detail. Therefore the event shape of a hadronic final state is studied depending on multiplicity of identified particles $(N)$, which is equal $N=N_{\text {tot }}$ for the exclusive approach and $N=N_{\text {ch }}$ in other cases.

Average values of main collective variables are presented at Fig 5 depend on multiplicity of the identified secondary particles in event. Experimental data are taken from $[22,23,48,56,63,67,69-71,90]$. At $\langle W\rangle \geq 4.0 \mathrm{GeV}$ right points correspond to integrated values $\langle S\rangle$ for a multiplicity range with low boundary indicated for $\langle W\rangle=$ 4.0 GeV namely (5a). We used the results for modified sphericity tensor with reduced momenta of final state particles [16] and sphericity definition from original papers for $K^{-} \mathrm{p}[22,69]$. Fit results of present work and corresponding curves at Fig 5 are described below. The intensification of spherical event shape is observed with final state multiplicity increasing for all types of interactions under study at intermediate energy domain $\sqrt{s}<60 \mathrm{GeV}$ (Fig 5). Rescattering of hadronic states in nuclear matter results in to the some increasing of $\langle S\rangle$ for $\pi^{-} \mathrm{Ne}$ in comparison with elementary reactions. Therefore one can see 
Table 8. Estimations for event shape observables*

\begin{tabular}{|c|c|c|c|c|c|c|}
\hline \multirow{2}{*}{$\begin{array}{l}\sqrt{s}, \\
\mathrm{TeV}\end{array}$} & \multicolumn{6}{|c|}{ Observable } \\
\hline & $\langle S\rangle$ & $\langle T\rangle$ & $\left\langle p_{\text {in }}^{2}\right\rangle,(\mathrm{GeV} / c)^{2}$ & $\left\langle p_{\text {out }}^{2}\right\rangle,(\mathrm{GeV} / c)^{2}$ & $\langle\mathrm{~A}\rangle$ & $\langle\mathrm{P}\rangle$ \\
\hline \multicolumn{7}{|c|}{$e^{+} e^{-}$annihilation } \\
\hline 0.5 & $0.050 \pm 0.002$ & $0.955 \pm 0.005$ & $0.9 \pm 0.8$ & $0.20 \pm 0.19$ & $0.026 \pm 0.002$ & $0.11 \pm 0.02$ \\
\hline 0.8 & $0.044 \pm 0.002$ & $0.959 \pm 0.005$ & $1.1 \pm 0.9$ & $0.23 \pm 0.23$ & $0.024 \pm 0.002$ & $-/ /-$ \\
\hline 1.0 & $0.042 \pm 0.002$ & $0.960 \pm 0.005$ & $1.2 \pm 1.0$ & $0.25 \pm 0.25$ & $0.023 \pm 0.002$ & $-1 /-$ \\
\hline 3.0 & $0.033 \pm 0.002$ & $0.966 \pm 0.004$ & $1.7 \pm 1.5$ & $0.35 \pm 0.37$ & $0.020 \pm 0.002$ & $-1 /-$ \\
\hline 5.0 & $0.031 \pm 0.002$ & $0.968 \pm 0.004$ & $2.0 \pm 1.8$ & $0.40 \pm 0.44$ & $0.019 \pm 0.002$ & $-/ /-$ \\
\hline \multicolumn{7}{|c|}{ ep interactions } \\
\hline 0.8 & - & - & $0.25 \pm 0.25$ & $0.08 \pm 0.02$ & - & - \\
\hline 1.6 & $0.038 \pm 0.002$ & $0.963 \pm 0.005$ & $0.28 \pm 0.27$ & $0.08 \pm 0.02$ & $0.021 \pm 0.002$ & $0.11 \pm 0.02$ \\
\hline \multicolumn{7}{|c|}{ pp collisions } \\
\hline 0.2 & $0.067 \pm 0.003$ & $0.946 \pm 0.007$ & $0.17 \pm 0.16$ & $0.08 \pm 0.05$ & $0.033 \pm 0.002$ & $0.14 \pm 0.03$ \\
\hline 0.5 & - & - & $0.18 \pm 0.18$ & $0.10 \pm 0.07$ & - & - \\
\hline 7.0 & $0.029 \pm 0.002$ & $0.969 \pm 0.004$ & $0.21 \pm 0.20$ & $0.16 \pm 0.13$ & $0.019 \pm 0.002$ & $0.11 \pm 0.02$ \\
\hline 14 & $0.027 \pm 0.002$ & $0.971 \pm 0.004$ & $0.21 \pm 0.21$ & $0.17 \pm 0.15$ & $0.018 \pm 0.002$ & $-/ /-$ \\
\hline 28 & $0.025 \pm 0.002$ & $0.973 \pm 0.003$ & $0.22 \pm 0.21$ & $0.19 \pm 0.17$ & $-/ /-$ & $-/ /-$ \\
\hline 42 & $0.024 \pm 0.002$ & $0.974 \pm 0.003$ & $-/ /-$ & $0.20 \pm 0.18$ & $-/ /-$ & $-/ /-$ \\
\hline 100 & $0.023 \pm 0.002$ & $0.976 \pm 0.003$ & $0.22 \pm 0.22$ & $0.23 \pm 0.21$ & $-/ /-$ & $-/ /-$ \\
\hline 200 & $-/ /-$ & $0.977 \pm 0.003$ & $0.23 \pm 0.22$ & $0.25 \pm 0.23$ & $-/ /-$ & $-1 /-$ \\
\hline
\end{tabular}

${ }^{*}$ Fit results for joint data samples are used to estimate the $\langle S\rangle,\langle T\rangle,\langle\mathrm{A}\rangle$ and $\langle\mathrm{P}\rangle$ for a certain type of interaction, thus the some estimation is indicated once if various interactions are studied at the same energy.

the good agreement of results obtained for $\pi^{-} \mathrm{Ne}$ and for $(\bar{\nu}+\nu) \mathrm{p}$ in despite of some difference in the initial energies. Similar accordance with general tendency is observed for $\pi^{-} \mathrm{p}, K^{+} \mathrm{p}, \overline{\mathrm{p}} \mathrm{p}$ and $(\bar{\nu}+\nu) \mathrm{p}$ interactions at larger initial energies too. In [15] analytical dependence $\langle S\rangle(N) \propto N^{-0.5}$ was derived for isotropic distribution of secondary particles in phase space for two ultimate cases: non-relativistic and extremely relativistic ones which are shown at Fig 5 a by dotted thin curves "1" and "2" respectively. Results for $\pi^{-} \mathrm{Ne}$ and for $(\bar{\nu}+\nu) \mathrm{p}$ agree well with phenomenological dependence for ultra-relativistic case, that confirms the difficulty of separation of jet events at $\sqrt{s} \sim 3-5 \mathrm{GeV}$ on the basis of one event shape observable (2) for interactions of any types. Thus, one can suggest, that the universal estimation of the low energy boundary for experimental appearance of event jet structure in multiparticle production processes is $\sqrt{s_{c}} \sim 3 \mathrm{GeV}$. At increasing of initial energy one can see that estimations extracted for isotropic one-particle distribution are essentially larger than experimental values $\langle S\rangle$, that corresponds to an amplifying for appearance of event jet structure. Functional behavior of dependence $\langle T\rangle(N)$ in case of $e^{+} e^{-}$annihilation at significantly larger initial energy qualitatively distinguishes from behavior of $\langle T\rangle(N)$ for hadron-hadron collisions at $\sqrt{s}<12 \mathrm{GeV}$ but $\langle T\rangle$ decreases with multiplicity increasing for all interaction types under study (Fig $5 \mathrm{~b}$ ). One need to emphasize that universality of geometry of events can be considered as evidence for similarity of dynamics of soft hadronic jet production in various interactions in wide initial energy range. Therefore dependencies $\langle S\rangle(N)$ and $\langle T\rangle(N)$ indicate at possible experimental appearance of color degrees of freedom in hadronic jet production at $\sqrt{s} \sim 3-5 \mathrm{GeV}$.

Figure 6 shows the multiplicity dependence for average values of a square of in-plane transverse momentum (a), square of transverse momentum out of the event plane (b), alignment (c), and planarity (d). Estimation values for $\left\langle p_{\text {in }}^{2}\right\rangle$ and $\left\langle p_{\text {out }}^{2}\right\rangle$ in case of $\pi^{-}$p at $40 \mathrm{GeV} / c$ were calculated on the basis of experimental data for eigenvalues of tensor $S^{\alpha \beta}$ and for $N_{\mathrm{ch}}$ from [23]. For $\pi^{ \pm} \mathrm{p}$ interactions at initial momenta $4-25 \mathrm{GeV} / c$ values of alignment and planarity corresponded to definitions used in present work (Table 1) have been estimated on the basis of experimental results from [54].

One can see that behavior of $\left\langle p_{\text {in }}^{2}\right\rangle(N)$ (Fig $[a$ ) and $\left\langle p_{\text {out }}^{2}\right\rangle(N)$ (Fig. 6b) depends both on initial energy and from reaction type. Available experimental results for components of transverse momentum under study do not allow to make conclusions concerning presence and character of the universal analytical dependence describing experimental data for different interactions and / or initial energies. But one can conclude that hadronic interactions show the decreasing of $\left\langle p_{\text {in }}^{2}\right\rangle(N)$ and increasing of $\left\langle p_{\text {out }}^{2}\right\rangle(N)$ with multiplicity increasing in general.

Parameters characterized of alignment (Fig $6 \mathrm{c}$ ) and planarity (Fig $[\mathrm{d})$ of event show smooth growth at multiplicity increasing for all types of interactions and initial energy domain under study, i.e. the events are less planar and are characterized smaller alignment at larger hadron multiplicity in final state. Decreasing of values of parameter $\langle\mathrm{A}\rangle$ is observed with increasing of beam momentum, i.e. the alignment of hadrons along the principal axis $\boldsymbol{n}_{1}$ 

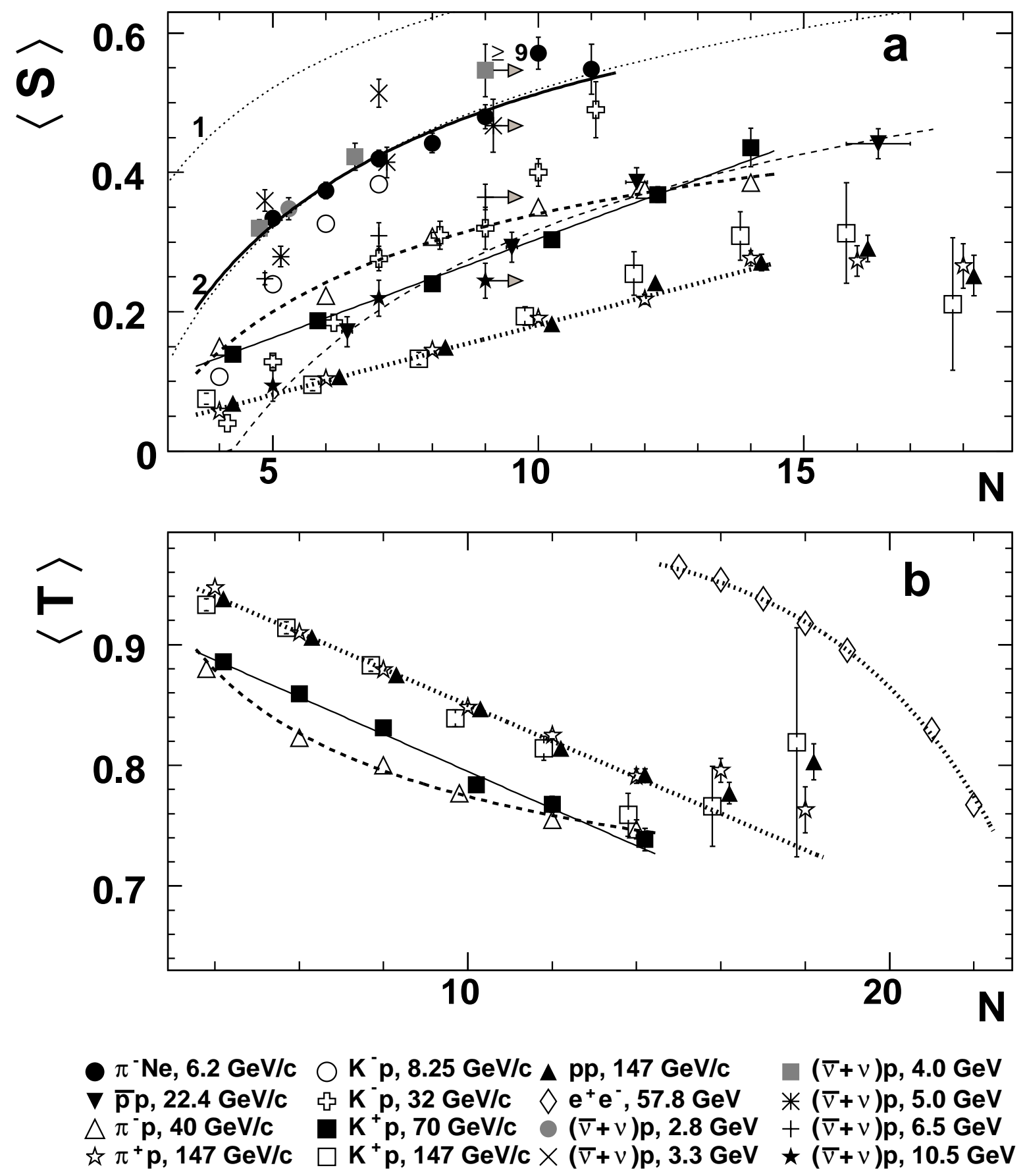

Fig. 5. Dependence of average values of sphericity (a) and thrust (b) on multiplicity of identified particles in event. Experimental results for $\pi^{-} \mathrm{Ne}$ are from [70, 71], for $K^{-} \mathrm{p}-$ from [22, 69], for $\pi^{-} \mathrm{p}$ at $40 \mathrm{GeV} / c-$ from [23], for $K^{+} \mathrm{p}$ at $70 \mathrm{GeV} / c-$ from [56], for $\overline{\mathrm{p}} \mathrm{p}$ at $22.4 \mathrm{GeV} / c-$ from [67], for $\mathrm{h}^{+} \mathrm{p}$ at $147 \mathrm{GeV} / c-$ from [63], for $(\bar{\nu}+\nu) \mathrm{p}$ - from [48]. Estimations for an average thrust values in case of $e^{+} e^{-}$are obtained on the basis of results from [90]. For $(\bar{\nu}+\nu)$ p average invariant mass of hadronic final state, $\langle W\rangle$, is indicated above, for $e^{+} e^{-}-$initial energy. Dotted thin lines marked as "1" and "2" at (a) are phenomenological approximations from [15]. Fit results from other publications, namely, for sphericity (a) and thrust (b) in $\mathrm{h}^{+} \mathrm{p}$ hadronic interactions from [63]; for $e^{+} e^{-}$annihilation for thrust (b) from [90], are shown by thick dotted lines. Statistical errors are shown. See text for explanation of fit curves in more detail. 

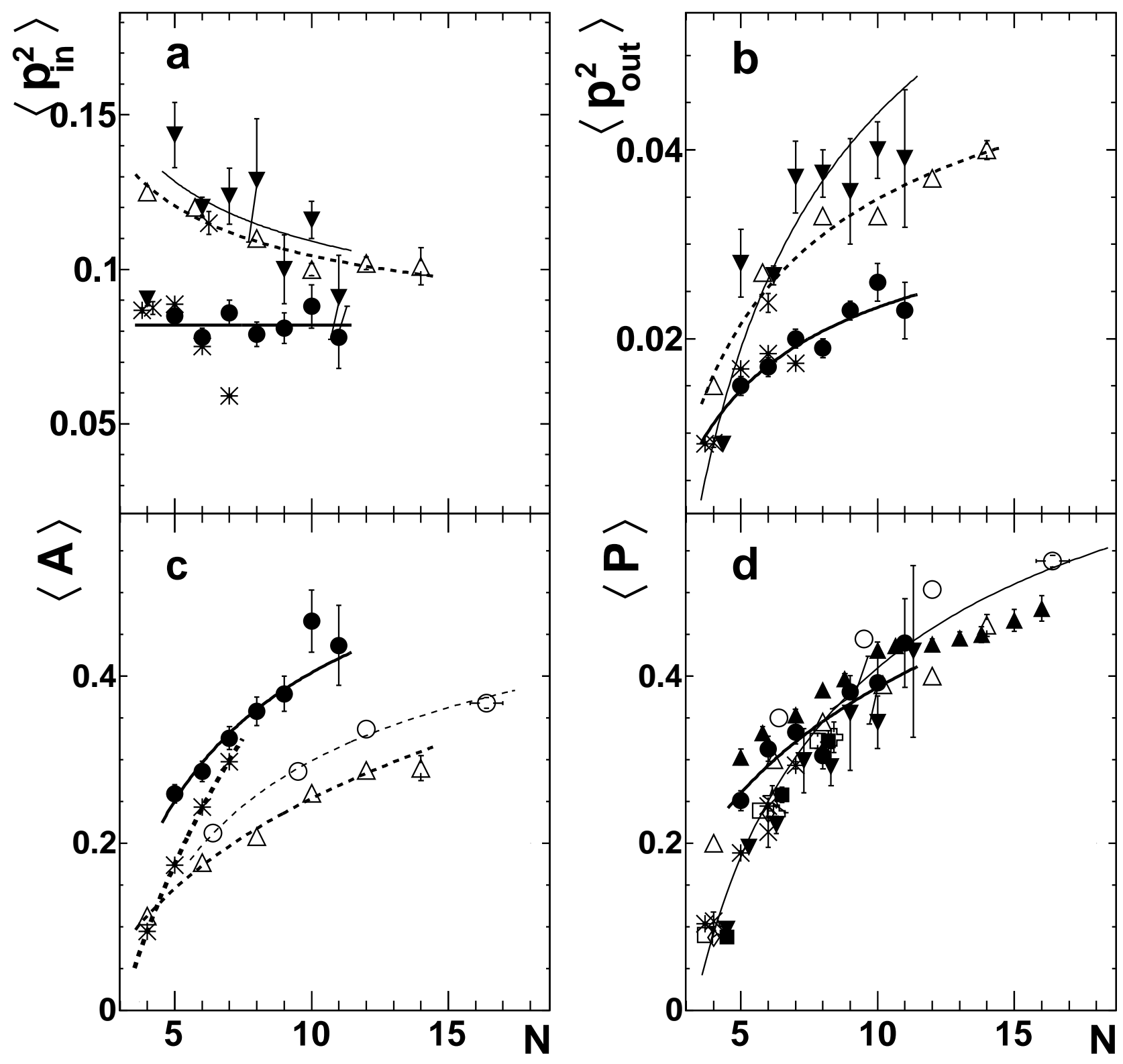

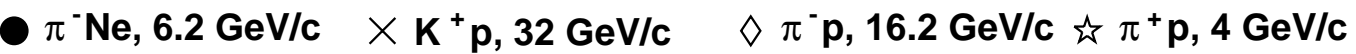
* K $\mathrm{K}^{-} \mathrm{p}, 8.25 \mathrm{GeV} / \mathrm{c} \bigcirc \overline{\mathrm{p}} \mathrm{p}, 22.4 \mathrm{GeV} / \mathrm{c}$ ↔ $\pi^{-} \mathrm{p}, 25 \mathrm{GeV} / \mathrm{c} \quad \pi^{+} \mathrm{p}, 8 \mathrm{GeV} / \mathrm{c}$ $\nabla \mathrm{K}$ p, $32 \mathrm{GeV} / \mathrm{c} \quad \Delta \mathrm{h}^{ \pm} \mathrm{Be}, 200 \mathrm{GeV} / \mathrm{c} \triangle \pi^{-p}, 40 \mathrm{GeV} / \mathrm{c} \quad \square \pi^{+} \mathrm{p}, 16 \mathrm{GeV} / \mathrm{c}$

Fig. 6. Average values of collective parameters describing the event shape in the "principal axes" coordinate system depend on multiplicity of the secondary identified particles: $\mathrm{a}-\left\langle p_{\text {in }}^{2}\right\rangle, \mathrm{b}-\left\langle p_{\text {out }}^{2}\right\rangle, \mathrm{c}-\langle\mathrm{A}\rangle, \mathrm{d}-\langle\mathrm{P}\rangle$. Values of $\left\langle p_{\text {in }}^{2}\right\rangle$ and $\left\langle p_{\text {out }}^{2}\right\rangle$ are shown in $(\mathrm{GeV} / c)^{2}$. Results for $\pi^{-} \mathrm{Ne}$ are from [70, 71], for $\pi^{-} \mathrm{p}$ at $16,25 \mathrm{GeV} / c$ and for $\pi^{+} \mathrm{p}$ at $4,8,16 \mathrm{GeV} / c$ are from [54], for $K^{-} \mathrm{p}$ - from [22, 69], for $\bar{p} p$ at $22.4 \mathrm{GeV} / c$ - from [67], for $K^{+}$p at $32 \mathrm{GeV} / c-$ from [68], for $\pi^{-}$p at $40 \mathrm{GeV} / c-$ from [23], for $\mathrm{h}^{ \pm} \mathrm{Be}$ at $200 \mathrm{GeV} / c$ - from [73]. Statistical errors are shown. See text for explanation of the curves. 
Table 9. Fit results for multiplicity dependence in hadronic interactions

\begin{tabular}{lcccc}
\hline Sample & \multicolumn{5}{c}{ Fit parameter } \\
\cline { 2 - 5 } & $a_{1}$ & $a_{2}$ & $a_{3}$ (fixed) & $\chi^{2} / \mathrm{ndf}$ \\
\hline \multicolumn{5}{c}{$\langle S\rangle$} \\
\hline$\pi^{-} \mathrm{Ne}$ & $0.97 \pm 0.05$ & $-1.44 \pm 0.13$ & -0.5 & 1.89 \\
$\overline{\mathrm{p}} \mathrm{p}$ & $0.91 \pm 0.07$ & $-1.9 \pm 0.2$ & $-/ /-$ & 0.41 \\
$\pi^{-} \mathrm{p}$ & $0.680 \pm 0.009$ & $-1.07 \pm 0.03$ & $-/ /-$ & 6.33 \\
$K^{+} \mathrm{p}$ & $0.021 \pm 0.009$ & $0.028 \pm 0.001$ & 1.0 & 0.63 \\
\hline \multicolumn{5}{c}{$\langle T\rangle$} \\
\hline$\pi^{-} \mathrm{p}$ & $0.594 \pm 0.015$ & $0.57 \pm 0.04$ & -0.5 & 0.22 \\
$K^{+} \mathrm{p}$ & $0.949 \pm 0.005$ & $-0.015 \pm 0.001$ & 1.0 & 1.94 \\
\hline
\end{tabular}

(jet axis) is amplified for higher initial energies. Influence of strange particles in a final state leads to small values of alignment in case of $K^{-} \mathrm{p}$ interactions at initial momentum 8.25 GeV/c [22] at small multiplicities. Moreover estimations of alignment parameter for $K^{ \pm}$p at $32 \mathrm{GeV} / c$ $[68,69]$ are smaller significantly in the case of sphericity tensor definition (4) than that for other experiments at close energies. Comparison of Fig 6c and Fig 6] demonstrates, that the parameter $\langle\mathrm{P}\rangle$ is less sensitive to presence of strange particles in final state, than alignment. Detail additional study shows that behaviors of dependencies at Fig 5 and at Fig, 6 are similar for total and charged multiplicities and the general picture changes insignificantly at substitution $N_{\text {tot }}$ on $N_{\text {ch }}$ and vase versa.

The situation with theoretical description of behavior of multiplicity dependence for collective parameters differs from the situation for energy dependence of some observables, at least. Apparently, there is no theoretical or phenomenological predictions for multiplicity dependence beyond [15] or experimental data fits for main collective variables $[63,90]$. Taking into account monotonic and smooth behavior of dependencies under study, experimental data for average values of collective parameters in hadronic and (anti)neutrino interactions have been fitted by general power function (7) for $Y \equiv S, T, p_{\text {in }}^{2}, p_{\text {out }}^{2}, \mathrm{~A}, \mathrm{P}$ and $X \equiv N$ in the case considered in this section. Detail study of fit results for various data ensembles for all collective observables has shown, that better fit quality is achieved, usually, for a special case of (7) at the following fixed values $a_{3}=-0.5$ or $a_{3}=1.0$. The first case agrees with function suggested in [15] for sphericity. Approximations are shown by thick solid line for $\pi^{-}$Ne and by thin dashed line for $\bar{p}$ p at $22.4 \mathrm{GeV} / c$ on Fig 5 a ; by thick dashed line for $\pi^{-} \mathrm{p}$ and by thin solid line for $K^{+} \mathrm{p}$ at $70 \mathrm{GeV} / c$ on Fig 5 . Results for fit by function (7) at fixed $a_{3}$ are shown in Table 9 for hadronic interactions under study. As seen, fit quality are acceptable for most data samples. One need to emphasize that general function (77) allows to get the best quality for $K^{+} \mathrm{p}$ collisions $\left(\chi^{2} / \mathrm{ndf}=0.01\right)$ with the following values of fit parameters: $a_{1}=0.08 \pm 0.03, a_{2}=0.009 \pm 0.006, a_{3}=1.4 \pm 0.3$. But the $a_{3}$ value is close to unit within errors and this general approximation agrees quite reasonable with linear one. As indicated above, modified both sphericity tensor and definition of corresponding observable from original papers which slightly differs from definition indicated in Table 1 are used for $K^{-}$p interactions. Reducing of influence of leading particles results in some faster intensification of spherical event shape with multiplicity increasing in comparison with behavior of $\langle S\rangle(N)$ dependence for other hadronic interactions at close energies for sphericity tensor definition (4). Otherwise, results for $K^{-}$p interactions at $8.25 \mathrm{GeV} / c$ and $32 \mathrm{GeV} / c$ agree with other experimental points qualitatively but for the first case corresponding fit quality is statistical unacceptable due to, possibly, small experimental errors. The following results have been obtained for fit of $\langle S\rangle(N)$ for $K^{-} \mathrm{p}$ collisions at $32 \mathrm{GeV} / c$ by (7): $a_{1}=-0.94 \pm 0.01, a_{2}=0.608 \pm 0.009$, $a_{3}=0.346 \pm 0.006$ at $\chi^{2} / \mathrm{ndf}=1.33$ (we do not show the curve at Fig 5 a for more clearly picture). Multiplicity dependence of sphericity for (anti)neutrino beams are fitted by function (7) with fixed $a_{3}=-0.5$ at all energies with statistically reasonable qualities. Results of fit for $(\bar{\nu}+\nu)$ p interactions are presented in Table 10 (we do not show corresponding curves at Fig 5 a for more clearly picture).

Experimental data for various interaction shown at Fig 5 and fit results for main collective variables (Tables 9 and 10) allow to suggest that intensification of spherical event shape with multiplicity increasing seems more powerful in energy domain $\sqrt{s}>11 \mathrm{GeV}$ than that at lower initial energies.

Table 10. Fit results for multiplicity dependence of $\langle S\rangle$ at (anti)neutrino beams

\begin{tabular}{lccc}
\hline$\langle W\rangle, \mathrm{GeV}$ & \multicolumn{3}{c}{ Fit parameter } \\
\cline { 2 - 4 } & $a_{1}$ & $a_{2}$ & $\chi^{2} / \mathrm{ndf}$ \\
\hline 4.0 & $1.09 \pm 0.11$ & $-1.7 \pm 0.3$ & 1.34 \\
5.0 & $1.08 \pm 0.12$ & $-1.8 \pm 0.3$ & 0.42 \\
6.5 & $0.69 \pm 0.07$ & $-1.0 \pm 0.2$ & 0.19 \\
10.5 & $0.72 \pm 0.11$ & $-1.4 \pm 0.3$ & 1.22 \\
\hline
\end{tabular}

Notations for approximation curves at Fig 6 are the same as well as on Fig $[5$ for corresponding reactions. More- 
Table 11. Values of fit parameters for function (7)

\begin{tabular}{lccc}
\hline \multirow{1}{*}{ Data } & \multicolumn{3}{c}{ Fit parameter } \\
\cline { 2 - 4 } sample & $a_{1}$ & $a_{2}$ & $\chi^{2} / \mathrm{ndf}$ \\
\hline \multicolumn{4}{c}{$\left\langle p_{\text {in }}^{2}\right\rangle$} \\
\hline$\pi^{-} \mathrm{Ne}$ & $0.082 \pm 0.002$ & 0.0 (fixed) & 0.88 \\
$K^{-} \mathrm{p}$ & $0.06 \pm 0.02$ & $0.15 \pm 0.06$ & 1.47 \\
$\pi^{-} \mathrm{p}$ & $0.065 \pm 0.004$ & $0.123 \pm 0.012$ & 2.78 \\
\hline \multicolumn{5}{c}{$\left\langle p_{\text {out }}^{2}\right\rangle$} \\
\hline$\pi^{-} \mathrm{Ne}$ & $0.045 \pm 0.004$ & $-0.07 \pm 0.01$ \\
$K^{-} \mathrm{p}$ & $0.104 \pm 0.004$ & $-0.190 \pm 0.008$ & 1.36 \\
$\pi^{-} \mathrm{p}$ & $0.067 \pm 0.002$ & $-0.102 \pm 0.005$ & 2.82 \\
\hline \multicolumn{5}{c}{$\langle\mathrm{A}\rangle$} \\
\hline$\pi^{-} \mathrm{Ne}$ & $0.77 \pm 0.06$ & $-1.17 \pm 0.14$ & 0.89 \\
$\overline{\mathrm{p} p}$ & $0.65 \pm 0.01$ & $-1.10 \pm 0.03$ & 3.96 \\
$\pi^{-} \mathrm{p}$ & $0.50 \pm 0.01$ & $-0.79 \pm 0.03$ & 6.15 \\
\hline \multicolumn{5}{c}{$\langle\mathrm{P}\rangle$} \\
\hline$\pi^{-} \mathrm{Ne}$ & $0.68 \pm 0.06$ & $-0.94 \pm 0.15$ & 2.06 \\
$K_{\text {-beams }}$ & $0.885 \pm 0.013$ & $-1.56 \pm 0.03$ & 2.47 \\
$\pi$-beams & $0.912 \pm 0.010$ & $-1.64 \pm 0.02$ & 8.13 \\
\hline
\end{tabular}

over the fit curve for $K^{-} \mathrm{p}$ at $32 \mathrm{GeV} / c$ is shown by solid thin line on Fig 6 a, b; for $K^{-}$p at $8.25 \mathrm{GeV} / c$ - by dotted line at Fig 6r; ; and curve for all available hadronic interactions is demonstrated by thin solid line at Fig 6 d. For multiplicity dependence of alignment in $K^{-}$p reaction at $8.25 \mathrm{GeV} / c$ the following values of fit parameters have been obtained for (7): $a_{1}=-3.035 \pm 0.008, a_{2}=$ $2.676 \pm 0.008, a_{3}=0.113 \pm 0.001$, and $\chi^{2} / \mathrm{ndf}=1.55$. Fit result for various experimental data samples by specific case of function (7) at fixed $a_{3}=-0.5$ are shown in Table 11 for all interactions and event shape variables with the exception of $\left\langle p_{\text {in }}^{2}\right\rangle(N)$ for $\pi^{-} \mathrm{Ne}$ at $6.2 \mathrm{GeV} / c$ discussed below. As seen, multiplicity dependence for all collective variables are fitted by specific case of (7) with reasonable quality in the case of $\pi^{-}$Ne hadron-nuclear collisions. For this reaction dependence of $\left\langle p_{\text {in }}^{2}\right\rangle$ on $N$ (Fig 6 ) is fitted with best quality by the simplest case of (7) which corresponds to constant. On the other hand fit by functional form (7) with fixed $a_{3}=-0.5$ shows reasonable and only slightly poorer quality $\left(\chi^{2} / \mathrm{ndf}=1.02\right)$. Thus the behavior of $\left\langle p_{\text {in }}^{2}\right\rangle(N)$ does not contradict with specific case of function (17) indicated above for hadron-nuclear reaction under study. The reasonable quality has been obtained for fit of data sample for $\left\langle p_{\text {in }}^{2}\right\rangle$ in $K^{-}$p at $32 \mathrm{GeV} / c$ without first point only. Dependencies of $\left\langle p_{\text {in }}^{2}\right\rangle$ and $\left\langle p_{\text {out }}^{2}\right\rangle$ on $N$ shown for $K^{-}$p at $8.25 \mathrm{GeV} / c$ on Fig 6 and Fig 6 , respectively, agree with approximation function (7) at $a_{3}=-0.5$ qualitatively but the corresponding fit qualities are statistical unacceptable for this reaction due to dispersion of experimental points and, possibly, small errors. As seen from Table 11, planarity results obtained in interactions with kaon beams are fitted by (7) with reasonable $\chi^{2} /$ ndf. Experimental results for pion beams agree with approx- imation function (7) qualitatively only and fit quality is some poorer for this incoming particle than that for kaon beams. The approximation curves for two beam types under study are very close to each other, moreover results for all hadronic interactions demonstrate similar values for planarity parameter $\langle P\rangle$ at corresponding multiplicities (Fig 6 d). Thus we have attempted to fit all available experimental points by (7) at fixed $a_{3}=-0.5$. One can conclude from Fig 6 that total sample of experimental results for hadronic interactions agrees with specific case of function (7) at qualitative level but the fit quality is statistically unacceptable $\left(\chi^{2} /\right.$ ndf $\left.\simeq 20\right)$.

Additional investigations show the following. First of all, power function (7) and it's special case at fixed $a_{3}=$ -0.5 describe dependencies of collective variables on charged multiplicity reasonable at qualitative level too. Second, as expected, using of modified sphericity tensor with reduced momenta of final state particles for $K^{-} \mathrm{p}[22,69]$ result in growth of values of $\langle\mathrm{A}\rangle$ (especially) and $\langle\mathrm{P}\rangle$ with the preservation of functional form for corresponding dependencies. On the other hand the behavior of dependence of components of particle transverse momentum in and out of the event plane on multiplicity changes dramatically, namely, $\left\langle p_{\text {in }}^{2}\right\rangle$ and $\left\langle p_{\text {out }}^{2}\right\rangle$ are shown the power growth with $N$ increasing and significantly larger values than that for sphericity tensor definition (4).

\section{Summary}

In the framework of present paper the database of experimental results for traditional event shape variables was created for wide set of interaction types. This database is used for investigation of dependencies of collective parameters on initial energy and multiplicity in various interactions and for joint samples.

At intermediate energies influence of nuclear matter, in particular, multinucleon interactions leads to significantly more isotropic distribution of secondary particles for hadron-nuclear reactions in comparison with hadronhadron ones, to growth of transverse momentum, attenuation of alignment and planarity of events. Leading particles result in the inverse influence on event shape in hadronic reactions in comparison with intranuclear rescattering.

Energy dependencies of the main collective observables sphericity and thrust show universal behavior for various interactions. Phenomenological models based on the QCD, in particular, dispersive model allow to describe $\langle T\rangle(s)$ in wide range of initial energies down to strongly non-perturbative domain $\sqrt{s} \sim 2-3 \mathrm{GeV}$ at qualitative level at least. Approximation of energy dependence of average thrust values for various interactions allows to extract $\alpha_{S}\left(M_{Z}\right)$ estimations which are in agreement both with world average value and with results obtained by other methods. Values of transverse momentum components which are in / out of event plane for $e^{+} e^{-}$interactions increase faster with growth of $\sqrt{s}$, than that for $e \mathrm{p}$ and hadronic interactions. Energy dependencies of alignment and planarity suppose the descriptions by universal 
empirical functions for different interaction types. Using suggested analytic approximation functions estimations of values of collective parameters under study have been obtained for present and future collider-research facilities. At $\sqrt{s} \sim 5-7 \mathrm{TeV}$ average values of collective variables studied in the present paper do not depend on initial energy within errors. Thus, these values of $\sqrt{s}$ can be considered as an estimation of the low bound of asymptotic region for traditional collective parameters.

Spherical event shape is amplified with multiplicity increasing and one can suggest, that the universal estimation of the low energy boundary for experimental appearance of event jet structure in multiparticle production processes is $\sqrt{s_{c}} \sim 3 \mathrm{GeV}$. Usually, multiplicity dependence of collective variables under study agree with power function in energy domain $\sqrt{s}<12 \mathrm{GeV}$ at qualitative level at least.

\section{References}

1. C.W. Bauer et al., Phys. Rev. D 78, 034027 (2008).

2. Particle data group: K. Nakamura et al., J. Phys. G 37, 1 (2010).

3. S. Moretti et al., J. High Energy Phys. 9808, 001 (1998).

4. G.P. Salam, arXiv: 0906.1833 [hep-ph].

5. S.D. Ellis et al., Prog. in Part. Nucl. Phys. 60, 484 (2008).

6. G. Sterman, S. Weinberg, Phys. Rev. Lett. 39, 1436 (1977).

7. E. Gardi, J. Rathsman, Nucl. Phys. B 638, 243 (2002).

8. T. Kluge, Eur. Phys. J. C 33, s416 (2004).

9. P. Konar, P. Roy, Phys. Lett. B 634, 295 (2006).

10. P.M. Chesler et al., Phys. Rev. D 79, 025021 (2009).

11. M.V. Savina, Phys. At. Nucl. 74, 496 (2011).

12. J.D. Bjorken, S.J. Brodsky, Phys. Rev. D 1, 1416 (1970).

13. G. Hanson et al., Phys. Rev. Lett. 35, 1609 (1975).

14. M.J. Counihan, Phys. Lett. B 59, 365 (1975).

15. P. Grassberger, E.H. De Groot, Nucl. Phys. B 102, 297 (1976).

16. G. Parisi, Phys. Lett. B 74, 65 (1978).

17. PLUTO Collaboration: Ch. Berger et al., Z. Phys. C 12, 297 (1982).

18. S. Brandt et al., Phys. Lett. 12, 57 (1964).

19. E. Farhi, Phys. Rev. Lett. 39, 1587 (1977).

20. S. Brandt, H.D. Dahmen, Z. Phys. C 1, 61 (1979).

21. AMY Collaboration: Y.K. Li et al., Phys. Rev. D 41, 2675 (1990).

22. ADLV Collaboration: M. Kakoulidou et al., Nucl. Phys. B 130, 189 (1977).

23. V.G. Grishin et al., Sov. J. Nucl. Phys. 37, 545 (1983).

24. P. Darriulat, Ann. Rev. Nucl. Part. Sci. 30, 159 (1980).

25. The Durham HEP Reaction Data Databases (UK) http://durpdg.dur.ac.uk/hepdata/reac.html

26. DELCO Collaboration: M. Sakuda et al., Phys. Lett. B 152, 399 (1985).

27. HRS Collaboration: D. Bender et al., Phys. Rev. D 31, 1 (1985).

28. Mark II Collaboration: A. Petersen et al., Phys. Rev. D 37, 1 (1988).

29. PLUTO Collaboration: Ch. Berger et al., Phys. Lett. B 78, 176 (1978); 82, 449 (1979).

30. DHHM Collaboration: F.H. Heimlich et al., Phys. Lett. B 86, 399 (1979).
31. CELLO Collaboration: H.J. Behrend et al., Z. Phys. C 14, 189 (1982); Phys. Lett. B 193, 376 (1987).

32. JADE Collaboration: P.A. Movilla Fernández et al., Eur. Phys. J. C 1, 461 (1998).

33. JADE and OPAL Collaborations: P. Pfeifenschneider et al., Eur. Phys. J. C 17, 19 (2000).

34. C. Pahl et al., Eur. Phys. J. C 60, 181 (2009).

35. MARK-J Collaboration: D.P. Barber et al., Phys. Rev. Lett. 44, 1722 (1980).

36. MARK-J Collaboration: B. Adeva et al., Phys. Rev. Lett. 50, 799 (1983).

37. PLUTO Collaboration: Ch. Berger et al., Phys. Lett. B 81, 410 (1979); Phys. Lett. B 86, 418 (1979).

38. TASSO Collaboration: R. Brandelik et al., Phys. Lett. B 83, 261 (1979); 86, 243 (1979); 89, 418 (1980); 94, 437 (1980); 135, 243 (1984); M. Althoff et al., Z. Phys. C 22, 307 (1984); W. Braunschweig et al., ibid. 41, 359 (1989); 45, 11 (1989); 47, 187 (1990).

39. TASSO Collaboration: R. Brandelik et al., Z. Phys. C 4, 87 (1980).

40. TOPAZ Collaboration: K. Nagai et al., Phys. Lett. B 278, 506 (1992); Y. Ohnishi et al., ibid. 313, 475 (1993).

41. Mark II Collaboration: G.S. Abrams et al., Phys. Rev. Lett. 63, 1558 (1989).

42. SLD Collaboration: K. Abe et al., Phys. Rev. D 51, 962 (1995).

43. ALEPH Collaboration: D. Buskulic et al., Z. Phys. C 55, 209 (1992); 73, 409 (1997); A. Heister et al., Eur. Phys. J. C 35, 457 (2004).

44. DELPHI Collaboration: P. Aarnio et al., Phys. Lett. B 240, 271 (1990); P. Abreu et al., Z. Phys. C 73, 11 (1996); 73, 229 (1997); P. Abreu et al., Phys. Lett. B 456, 322 (1999); J. Abdallah et al., Eur. Phys. J. C 29, 285 (2003); 37, 1 (2004).

45. L3 Collaboration: B. Adeva et al., Z. Phys. C 55, 39 (1992); M. Acciarri et al., Phys. Lett. B 489, 65 (2000); P. Achard et al., Phys. Rep. 399, 71 (2004).

46. OPAL Collaboration: M.Z. Akrawy et al., Z. Phys. C 47, 505 (1990); P.D. Acton et al., ibid. 55, 1 (1992); G. Alexander et al., ibid. 72, 191 (1996); K. Ackerstaff et al., ibid. 75, 193 (1997); G. Abbiendi et al., Eur. Phys. J. C 16, 185 (2000); 40, 287 (2005).

47. ABCLOS Collaboration: K.W.J. Barnham et al., Phys. Lett. B 85, 300 (1979).

48. M. Derrick et al., Phys. Lett. B 88, 177 (1979).

49. H1 Collaboration: C. Adloff et al., Phys. Lett. B 406, 256 (1997).

50. H1 Collaboration: C. Adloff et al., Eur. Phys. J. C 1, 495 (1998).

51. H1 Collaboration: C. Adloff et al., Eur. Phys. J. C 14, 255 (2000).

52. H1 Collaboration: A. Aktas et al., Eur. Phys. J. C 46, 343 (2006).

53. ZEUS Collaboration: J. Breitweg et al., Phys. Lett. B 421, 368 (1998); S. Chekanov et al., Phys. Rev. D 65, 052001 (2002); S. Chekanov et al., Eur. Phys. J. C 27, 531 (2003); S. Chekanov et al., Nucl. Phys. B 767, 1 (2007).

54. P. Kostka et al., Nucl. Phys. B 86, 1 (1975).

55. ABCCLVW Collaboration: R. Göttgens et al., Nucl. Phys. B 178, 392 (1981).

56. BCGMNS Collaboration: M. Barth et al., Nucl. Phys. B 192, 289 (1981).

57. CMOR Collaboration: A.L.S. Angelis et al., Nucl. Phys. B 244, 1 (1984). 
58. NA5 Collaboration: C. De Marzo et al., Nucl. Phys. B 211, 375 (1983).

59. EHS-RCBC Collaboration: J.L. Bailly et al., Phys. Lett. B 206, 371 (1988).

60. A. Breakstone et al., Z. Phys. C 11, 203 (1981).

61. UA2 Collaboration: J.A. Appel et al., Phys. Lett. B 165, 441 (1985); J. Alitti et al., ibid. 268, 145 (1991).

62. CMS Collaboration: V.Khachatryan et al., Phys. Lett. B 699, 48 (2011).

63. IHS Consortium: D.H. Brick et al., Z. Phys. C 15, 1 (1982).

64. B. Brown et al., Phys. Rev. Lett. 49, 711 (1982).

65. CDF Collaboration: T. Aaltonen et al., arXiv: 1103.5143 [hep-ex].

66. V.G. Grishin et al., Sov. J. Nucl. Phys. 38, 581 (1983).

67. ADHMPT Collaboration: E.G. Boos et al., Sov. J. Nucl. Phys. 31, 197 (1980).

68. FUSSR and CUSSR Collaborations: I.V. Ajinenko et al., Nucl. Phys. B 135, 365 (1978).

69. FUSSR and CUSSR Collaborations: M.Yu. Bogolyubskii et al., Sov. J. Nucl. Phys. 32, 73 (1980).

70. V.I. Mikhailichenko et al., Preprint MEPhI 002-95, Moscow (1995).

71. V.A. Okorokov, PhD thesis. Moscow Engineering Physics Institute (1996).

72. V.G. Grishin et al., Sov. J. Nucl. Phys. 38, 410 (1983).

73. C. Bromberg et al., Nucl. Phys. B 134, 189 (1978).

74. M. Basile et al., Nuovo Cimento A 79, 1 (1984).

75. I.L. Kiselevich et al., Phys. At. Nucl. 57, 2140 (1994).

76. A. Banfi et al., J. High Energy Phys. 0007, 002 (2000).

77. A. Banfi et al., J. High Energy Phys. 0105, 040 (2001).

78. A. Banfi, G.P. Salam, G. Zanderighi, J. High Energy Phys. 0201, 018 (2002).

79. A. Gehrmann-De Ridder et al., J. High Energy Phys. 0905, 106 (2009).

80. Yu.L Dokshitzer, B.R. Webber, Phys. Lett. B 352, 451 (1995).

81. Yu.L. Dokshitzer et al., Nucl. Phys. B 469, 93 (1996).

82. E. Gardi, G. Grunberg, J. High Energy Phys. 9911, 016 (1999).

83. E. Gardi J. High Energy Phys. 0004, 030 (2000).

84. E. Gardi, J. Rathsman, Nucl. Phys. B 609, 123 (2001).

85. Yu. Dokshitzer et al., Nucl. Phys. B 511, 396 (1998).

86. B.R. Webber, Nucl. Phys. B (Proc. Suppl.) 71, 66 (1999).

87. C. Pahl et al., Eur. Phys. J. C 64, 533 (2009).

88. S. Bethke, Eur. Phys. J. C 64, 689 (2009).

89. PHENIX Collaboration: S.S. Adler et al., Phys. Rev. D 74, 072002 (2006).

90. K. Nakabayashi et al., Phys. Lett. B 413, 447 (1997). 